\title{
Opposing expression gradients of calcitonin-related polypeptide alpha (Calca/Cgrpa) and tyrosine hydroxylase (Th) in type II afferent neurons of the mouse cochlea
}

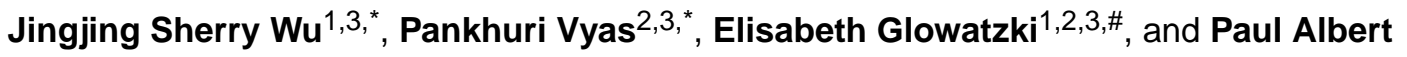 \\ Fuchs $\mathbf{s}^{1,2,3, \#}$ \\ ${ }^{1}$ Department of Neuroscience, The Johns Hopkins University School of Medicine, Baltimore, MD \\ 21205, USA \\ ${ }^{2}$ Department of Otolaryngology-Head and Neck Surgery, The Johns Hopkins University School of \\ Medicine, Baltimore, MD 21205, USA \\ ${ }^{3}$ The Center for Hearing and Balance and the Center for Sensory Biology, The Johns Hopkins \\ University School of Medicine, Baltimore, MD 21205, USA
}

\begin{abstract}
Type II spiral ganglion neurons (SGNs) are small caliber, unmyelinated afferents that extend dendritic arbors hundreds of microns along the cochlear spiral, contacting many outer hair cells (OHCs). Despite these many contacts, type II afferents are insensitive to sound and only weakly depolarized by glutamate release from OHCs. Recent studies suggest that type II afferents may be cochlear nociceptors, and can be excited by ATP released during tissue damage, by analogy to somatic pain-sensing $\mathrm{C}$-fibers. The present work compares the expression patterns among cochlear type II afferents of two genes found in C-fibers: calcitonin-related polypeptide alpha (Calca/ Cgrpa), specific to pain-sensing C-fibers, and tyrosine hydroxylase (Th), specific to low-threshold mechanoreceptive C-fibers, which was shown previously to be a selective biomarker of type II versus type I cochlear afferents (Vyas et al, 2016). Whole-mount cochlear preparations from 3week to 2-month-old CGRPa-EGFP(GENSAT) mice showed expression of Cgrpa in a subset of SGNs with type II-like peripheral dendrites extending beneath OHCs. Double labeling with other molecular markers confirmed that the labeled SGNs were neither type I SGNs nor olivocochlear efferents. Cgrpa starts to express in type II SGNs before hearing onset, but the expression level declines in the adult. The expression patterns of Cgrpa and $T h$ formed opposing gradients, with Th being preferentially expressed in apical and Cgrpa in basal type II afferent neurons, indicating
\end{abstract}

\footnotetext{
Address for correspondence: Elisabeth Glowatzki and Paul Albert Fuchs, The Johns Hopkins School of Medicine, Departments of Otolaryngology Head and Neck Surgery and Neuroscience, 720 Rutland Avenue, Ross 824, Baltimore MD 21205, USA, Tel.: 410-502-7008 (Glowatzki), 410-955-6311 (Fuchs), Fax: 410-614-4748, eglowat2@jhmi.edu, pfuchs1@jhmi.edu.

Both authors contributed equally

\#Both authors contributed equally

Author Contributions:

All authors had full access to all the data in the study and take responsibility for the integrity of the data and the accuracy of the data analysis. J.S.W. and P.V. designed, performed research and analyzed data; J.S.W., P.V., P.A.F. and E.G. discussed results and wrote the manuscript.

Conflict of Interest: The authors declare no competing financial interests.
} 
heterogeneity among type II afferent neurons. The expression of Th and Cgrpa was not mutually exclusive and co-expression could be observed, most abundantly in the middle cochlear turn.

\section{Keywords}

hair cell; hearing; spiral ganglion neuron; auditory nerve fiber; CGRP

\section{INTRODUCTION}

The mammalian cochlea contains two types of afferent neurons, distinguished by their morphology and innervation pattern. The majority (95\%) are larger diameter, myelinated type I afferents postsynaptic to single inner hair cells (IHCs). In contrast, a much smaller number of thinner, unmyelinated type II afferents extend dendrites hundreds of microns along the cochlear spiral, contacting many outer hair cells (OHCs). These distinct innervation patterns suggest distinct functional roles. Type I afferents transmit acoustic information, as proven by many decades of research (Young, 2008). The functional role of type II afferents is less certain. Their contribution to acoustic analysis is presumed to be minimal, due to their small number, relative insensitivity to sound (Brown, 1994; Robertson, 1984; Robertson, Sellick, \& Patuzzi, 1999) and weak excitation by OHC transmitter release (C. J. Weisz, Lehar, Hiel, Glowatzki, \& Fuchs, 2012). Instead, evidence suggests that type II afferents may signal cochlear damage (Flores et al., 2015; C. Liu, Glowatzki, \& Fuchs, 2015), and so are postulated to be 'cochlear nociceptors'.

Identifying genes that are differentially expressed in type II versus type I afferent neurons would enable genetic manipulation to isolate the effects of type II afferents from those of the much larger population of type I afferents. Understanding the cellular actions of these selectively expressed genes also could shed light on the overall function of type II afferents. In previous work, tyrosine hydroxylase (Th), a marker for C-type low threshold mechanoreceptors in the somatosensory system, was shown to express in lateral olivocochlear efferents and in type II but not type I afferent neurons, particularly in the apical cochlea (Vyas, Wu, Zimmerman, Fuchs, \& Glowatzki, 2016). This observation suggested a previously underappreciated heterogeneity among type II afferent neurons. Indeed, in contrast to the much diversified groups of ganglion neurons in other sensory systems such as vision and somatosensation (Abraira \& Ginty, 2013; Basbaum, Bautista, Scherrer, \& Julius, 2009; Sanes \& Masland, 2015), little is known beyond the type I and type II dichotomy for spiral ganglion neurons (SGNs) and activity differences among type I afferents. Therefore, the identification of additional genetic markers that could label type II afferent neurons in the basal cochlea is desirable. In view of the proposed role of type II afferents in detecting tissue damage, we continued to screen recombinant mouse lines labeling subpopulations of ganglion neurons in the somatosensory system, especially nociceptors, for their expression patterns in the cochlea.

One such gene, Calca, encoding for calcitonin-gene-related peptide alpha (CGRPa), is expressed in a wide variety of neuronal and non-neuronal cell types, ranging from keratinocytes to primary afferent neurons and spinal motor neurons (Russell, King, Smillie, 
Kodji, \& Brain, 2014). CGRP is a potent vasodilator and mediates neuronal-immune system communication (Assas, Pennock, \& Miyan, 2014). CGRP is expressed specifically in small $\mathrm{C}$ and $\mathrm{A} \delta$ neurons of dorsal root ganglia. The release of CGRP from sensory terminals is thought to intensify pain sensation, in part through its induction of vasodilation and inflammation, but also by facilitating central transmission. CGRP's most infamous role is in migraine pathogenesis, where again, a combination of effects on vasodilation, inflammation and central plasticity is thought to occur. CGRP has long been known to be expressed in cochlear efferent neurons (Kitajiri et al., 1985). Its involvement in nociception makes the expression in type II cochlear afferents shown here particularly interesting in the context of acoustic trauma and associated 'gain of function' pathologies such as hyperacusis.

\section{MATERIALS AND METHODS}

All procedures were in accordance with animal protocols approved by the Johns Hopkins University Animal Care and Use Committee. Mice of either sex were used in the experiments.

\section{Mouse lines}

The CGRPa-EGFP [Tg(Calca-EGFP)FG104Gsat/Mmucd; RRID:MMRRC_011187-UCD] mouse line, later referred to as CGRPa-EGFP(GENSAT) mouse line in this manuscript, was generated by random insertion of a bacterial artificial chromosome containing regulatory sequences of the Calca gene followed by the EGFP reporter gene. This mouse line was generated by GENSAT (Gong et al., 2003) and obtained for the study here on a mixed background from Dr. David Ginty (Harvard University, MA). The CGRPa-EGFP (GENSAT) mouse line has been previously validated by its expression pattern in dorsal root ganglion (DRG) neurons (Bai et al., 2015).

The CGRPaCreER $\left[\right.$ Calcat $\left.{ }^{\text {tm1.1(cre/ERT2)Ptch }}\right]$ mouse line was generated by insertion of the tamoxifen-inducible CreER gene into the Calca locus (Song et al., 2012). This mouse line was obtained from Dr. Jay Pasricha on a C57BL/6 background (Johns Hopkins Hospital, MD) with the permission of Dr. Pao-Tien Chuang (University of California, San Francisco, CA).

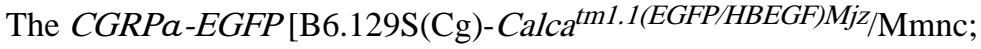
RRID:MMRRC_036773-UNC] mouse line, later referred to as CGRPa-EGFP(Zylka) mouse line in this manuscript, was generated by knocking-in a floxed GFP gene to the Calca locus (McCoy, Taylor-Blake, \& Zylka, 2012). Fixed cochlear tissue from this mouse line was provided by Dr. Eric McCoy (University of North Carolina, NC).

The $T h^{2 A-C r e E R}$ mouse line was generated by insertion of a $\mathrm{T}_{2} \mathrm{~A}$-peptide CreER cassette before the $3^{\prime}$ UTR of the Th gene using a recombineering protocol, allowing efficient transcription of both TH and Cre recombinase (Abraira et al., 2017).

Other mouse lines including Ai3 [B6.Cg-Gt(ROSA)26Sor ${ }^{\text {tm } 3(C A G-E Y F P) H z e / J}$,

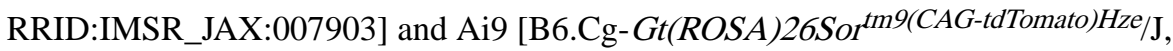
RRID:IMSR_JAX:007909] were obtained from the Jackson Laboratory (Bar Harbor, ME). 


\section{Immunofluorescence}

The cochleae from postnatal day (P) 5 to 2-month-old mice were harvested, perfused through the round and oval windows, and fixed for approximately $1 \mathrm{hr}$ at room temperature (RT) with 4\% paraformaldehyde (Electron Microscopy Sciences, Hatfield, PA) diluted in phosphate buffered saline (PBS). The whole mount neuroepithelia were microdissected in PBS. To better preserve and visualize the basal SGNs, some cochleae were decalcified in 0.2 $M$ ethylenediaminetetracetic acid (EDTA) in PBS for 1-2 days before further processing. To achieve better tissue penetration for certain primary antibodies, an additional step involving incubation in $30 \%$ sucrose, followed by a quick freeze at $-80{ }^{\circ} \mathrm{C}$ and thaw at $37{ }^{\circ} \mathrm{C}$ was performed. Dissected cochlear tissue was incubated for $\sim 1 \mathrm{hr}$ at RT in a blocking and permeabilizing buffer (1x PBS with $10 \%$ of normal donkey serum and $0.5 \%$ Triton X-100), before being incubated for $\sim 42 \mathrm{hrs}$ at RT with primary antibodies diluted in PBS containing $5 \%$ normal donkey serum, $0.25 \%$ Triton $\mathrm{X}-100$ and $0.01 \% \mathrm{NaN}_{3}$. Cochlear preparations were rinsed 3 times 5-10 min each in PBS before incubation with secondary antibodies (Table 2), diluted 1000 - 2000 times in PBS containing 5\% normal donkey serum and $0.25 \%$ Triton X-100 for 1-2 hrs at RT. Preparations were again rinsed 3 times 10-30 min each in PBS before mounting on glass slides in FluorSave mounting medium (Calbiochem, San Diego, CA). All rinses were performed on a rocking table at RT.

\section{Antibody Characterization}

All primary antibodies used in this study are listed in Table 1.

The rabbit anti-CGRP antibody used in this study labeled the well-established patterns of cochlear medial and lateral efferent terminals. Its immunolabeling could be completely abolished by pre-adsorption with rat $\alpha$-CGRP and partially eliminated with rat $\beta$-CGRP. Pre-adsorption with other types of neuropeptides resulted in no loss of immunostaining (manufacturer's datasheet). This antibody has been extensively used to label CGRP-positive DRG neurons and trigeminal sensory neurons (Hegarty, Tonsfeldt, Hermes, Helfand, \& Aicher, 2010; Li \& Ginty, 2014).

The rabbit anti-TH antibody used in this study labeled well the expected sympathetic fibers in the cochlea. It also showed co-extensive labeling with the knock-in $T^{2 A-C r e E R}$; Ai3 mouse line. It can detect the $\sim 60 \mathrm{kDa} \mathrm{TH}$ protein and has been validated for use in immunofluorescence applications (manufacturer's datasheet). This antibody has been used to label TH-positive neurons in mouse brain frozen sections (Du et al., 2001).

The goat anti-GFP antibody used in this study did not produce any labeling above background fluorescence on a wild-type cochlea, when this control tissue was processed together with the CGRPa-EGFP(GENSAT) mouse cochlea.

The rabbit anti-peripherin antibody has been validated for its use in immunohistochemistry. It stains a $\sim 57 \mathrm{kDa}$ band specifically in Western Blot analysis and does not stain vimentin, GFAP, a-internexin or any of the neurofilament subunits (manufacturer's datasheet). It has been used for labeling the type II SGNs in multiple previous publications (Flores-Otero \& Davis, 2011; Lang et al., 2011; McLean, Smith, Glowatzki, \& Pyott, 2009). 
The two antibodies against TuJ1 raised in rabbit and mouse have both been validated for multiple applications including Western Blot analysis, immunohistochemistry and immunofluorescence (manufacturer's datasheet). Both antibodies have been used extensively in previous publications, including some using cochlear samples (Davies, 2007; FloresOtero \& Davis, 2011).

The specificity of the mouse anti-NKAa3 antibody has been verified by the vendor using Western Blot analysis of canine skeletal muscle extracts. Its specific labeling of type I SGNs in rat cochlea has been well characterized (McLean et al., 2009).

The rabbit anti-dsRed antibody can recognize DsRed-Express, DsRed-Express2, DsRedMonomer, mCherry, DsRed2, E2-Crimson, tdTomato, mStrawberry, and mBanana, and both $\mathrm{N}$ - and C-terminal fusion proteins containing these fluorescent proteins in mammalian cell lysates. It specifically detects a band of $\sim 30-38 \mathrm{kDa}$ on Western Blot of lysates from HEK293 cells expressing DsRed-Express or DsRed-Monomer, but not for cells expressing AcGFP1(manufacturer's datasheet). In this study, this antibody is used to stain tdTomato protein expressed by transgenic mouse lines, similar use can be found in several publications (Chee, Pissios, \& Maratos-Flier, 2013; Hayes, Zhang, Albert, Zervas, \& Ahn, 2011; Ivanova, Lee, \& Pan, 2013).

The rabbit anti-myosin VI antibody and the mouse anti-myosin VIIa antibody are both used as hair cell markers and produced expected patterns (Korrapati, Roux, Glowatzki, \& Doetzlhofer, 2013; Roux et al., 2009). The guinea pig anti-VAChT and the mouse anti-SV2 are used to label cochlear efferent terminals, which produced the well-established pattern in the Organ of Corti (Kong, Adelman, \& Fuchs, 2008; S. F. Maison et al., 2010).

\section{Image acquisition and quantification}

Fluorescence images were acquired using a LSM 700 confocal microscope (Zeiss) with a Fluar 10×/0.50 M27 objective, a LCI Plan-Neofluar 25×/0.8 Imm Korr DIC M27 objective and a Fluar 40×/1.30 Oil M27 objective. Images were acquired in a $1024 \times 1024$ raster for each channel. Images are presented as maximum intensity z-projections through a subset of the collected optical stack. Brightness and contrast of confocal images were adjusted for better representation in some figures. These adjustments were performed using FiJi (RRID:SCR_002285) by changing the look up tables (LUTs), without any deconvolution, filtering, or gamma correction.

Quantification of the Th and Cgrpa-expressing SGNs was performed on confocal images acquired using a 10× objective. Each cochlea was microdissected into two or three pieces. Using a customized ImageJ plugin modified from Measure line (Eaton-Peabody Laboratories, Mass. Eye and Ear Infirmary), the location of every $10^{\text {th }}$ percentage of the whole cochlear length was identified. The SGN region of each cochlea was then divided into 10 parts by vertical lines to the tangent of each $10^{\text {th }}$ percentage of the cochlear length (Figure $5 \mathrm{~b}$ ). Cells in each bin were identified and labeled manually by carefully scanning through the confocal image slices in Zen software (Zeiss) to best visualize densely clustered SGNs. 


\section{RESULTS}

\section{Cgrpa is expressed in a subset of SGNs}

Calcitonin-related polypeptide alpha (Calca/Cgrpa) gene expression was examined in the cochlea of multiple mouse lines (see MATERIALS AND METHODS) and by immunolabeling of the native peptide.

EGFP expression driven by $C g r p a$ regulatory sequences in a BAC transgenic CGRPa-EGFP (GENSAT) mouse line was visualized by anti-GFP immunolabeling in cochlear wholemounts excised from 3-week to-2-month old mice. Consistent with the previous reports of CGRP immunolabeling in cochlear efferents (Cabanillas \& Luebke, 2002; Kitajiri et al., 1985; S. F. Maison, Adams, \& Liberman, 2003; Safieddine \& Eybalin, 1992; SliwinskaKowalska, Parakkal, Schneider, \& Fex, 1989), expression was observed in both medial (beneath OHCs, Figure 1 a, b-d) and lateral efferent fibers (beneath IHCs, Figure 1 a, e-g) throughout the cochlea. The terminal boutons of $\mathrm{GFP}^{+}$fibers were co-labeled with a cholinergic biomarker, the vesicular acetylcholine transporter (VAChT), in the $\mathrm{OHC}$ region (Figure $1 \mathrm{~d}$ and insets) and with the synaptic vesicle associated protein 2 (SV2) underneath the IHC region (Figure $1 \mathrm{G}$ and insets), confirming their medial and lateral efferent identities. Thus, the CGRPa-EGFP(GENSAT) mouse line could reliably report the Cgrpa promoter activity in cochlear efferents. Besides cochlear efferents, some spiral ganglion neurons (SGNs) were also $\mathrm{GFP}^{+}$(arrowheads, Figure $1 \mathrm{a}$ ), suggesting that CGRPa is expressed in a subset of SGNs. In another knock-in mouse line, CGRPaCreER; Ai3, labeling of SGNs could also be observed after heavy dose of tamoxifen injection (data not shown). However, the expression is possibly too low to produce enough CreER protein for reliable recombination of most of the SGNs in this mouse line.

\section{Young adult CGRPa-EGFP (GENSAT) cochleas label type II, but not type I SGNs}

The identity of labeled SGNs in 3-week to 2-month old CGRPa-EGFP(GENSAT) cochleas was explored by comparison with known markers of type I afferent neurons (Figure 2 a-f). $\beta$-Tubulin 3 (TuJ1) immunolabels all SGNs at perinatal ages. Starting around the first postnatal week, the TuJ1 immunolabeling declines in the cytoplasm of type II SGNs in various species (Barclay, Ryan, \& Housley, 2011; Lallemend et al., 2007; W. Liu, Bostrom, \& Rask-Andersen, 2009; W. Liu, Kinnefors, Bostrom, \& Rask-Andersen, 2010; Xing et al., 2012). Two TuJ1 antibodies were used to immunolabel the CGRPa-EGFP(GENSAT) cochleas (see Materials and Methods). The rabbit anti-TuJ1 antibody showed no or relatively weaker labeling of the $\mathrm{GFP}^{+}$SGNs ( $\mathrm{n}=2$ separate experiments; $28 \mathrm{GFP}^{+}$SGNs identified; total 109 SGNs analyzed, $11 \mathrm{GFP}^{+}$SGNs overlapped with TuJ1 staining). The mouse antiTuJ1 antibody showed no overlap labeling with $\mathrm{GFP}^{+}$SGNs ( $\mathrm{n}=3$ separate experiments; 23 $\mathrm{GFP}^{+}$SGNs identified; total 94 SGNs analyzed), suggesting their type II identity (Figure 2 a-c). a $3 \mathrm{Na}^{+} / \mathrm{K}^{+}$ATPase (NaKAa3) is a more stringent marker that labels the plasma membrane of type I afferent neurons (McLean et al., 2009). $\mathrm{GFP}^{+}$SGNs were negative for antibody labeling against NaKAa3 $\left(n=3\right.$ separate experiments; $22 \mathrm{GFP}^{+}$SGNs; total 81 SGNs analyzed) (Figure 2 d-f), again suggesting their type II identity. Furthermore, type IIlike morphology of processes and terminal boutons on OHCs could clearly be visualized in some regions of the CGRPa-EGFP(GENSAT) cochlea, when occasionally a low density of 
medial efferents was present in the base (Figure $2 \mathrm{~g}$ ). The fibers that run parallel to the OHC rows and the thin dendritic branches with a small terminal bouton are characteristic of type II fiber morphology that are clearly distinctive from the medial efferent terminals (Figure 1 b-d) (Martinez-Monedero et al., 2016; Vyas et al., 2016).

\section{Cgrpa is expressed in type II SGNs before hearing onset}

Type II, but not type I, afferents are labeled by antibodies against peripherin (Hafidi, 1998; Hafidi, Despres, \& Romand, 1993). GFP ${ }^{+}$SGNs in one-week-old CGRPa-EGFP (GENSAT) cochleas overlapped partially with peripherin immunolabeling (Figure 3 ). The extent of this overlap varied in different preparations, as shown in Figure 3. Only a fraction of $\mathrm{GFP}^{+}$neurons appeared to express peripherin in the example tissue in Figure $3 \mathrm{a}-\mathrm{c}$, however, virtually all $\mathrm{GFP}^{+}$SGNs were peripherin-positive in the tissue shown in Figure 3 d-f. Co-labeling with GFP and peripherin could also be seen in peripheral processes crossing the tunnel of Corti and extending along the outer spiral bundle external to the $\mathrm{OHC}$ rows (Figure $3 \mathrm{~g}-\mathrm{i}$ ). Some of these co-labeled peripheral processes could clearly be identified as type II fibers based on their fiber executing a sharp turn towards the cochlear base in the OHC region. Peripherin ${ }^{+}, \mathrm{GFP}^{-}$SGNs are presumably type II afferent neurons that do not express Cgrpa. As will be explained later, this could be due to the variable expression level of Cgrpa along the cochlear spiral. The identity of peripherin', $\mathrm{GFP}^{+} \mathrm{SGNs}$ is uncertain. Recent work suggested that peripherin immunolabels only a subset of type II SGNs (S. Maison, Liberman, \& Liberman, 2016; Nishimura, Noda, \& Dabdoub, 2017; Vyas et al., 2016). Therefore, some of the peripherin ${ }^{-}, \mathrm{GFP}^{+}$SGNs are most likely type II afferent neurons. However, as a much larger number of $\mathrm{GFP}^{+} \mathrm{SGNs}$ was found in one-week-old CGRPa-EGFP(GENSAT) cochleas (Figure $5 \mathrm{~d}$ ) compared to older ages, it is possible that some of the young $\mathrm{GFP}^{+}$SGNs are type I afferent neurons, given that the developmental loss of type II SGNs in rodents is usually completed within the first postnatal week (Barclay et al., 2011; Echteler \& Nofsinger, 2000; Rueda, de la Sen, Juiz, \& Merchan, 1987).

Two additional experiments confirmed the expression of Cgrpa in type II afferent neurons. Antibodies against CGRP labeled outer spiral bundles (OSBs) in the apical turn of a P6 wild type mouse cochlea, corresponding to type II afferent fiber bundles (Figure 4 a). CGRP antibody also labeled a dense plexus beneath IHCs, corresponding to lateral olivocochlear (LOC) efferent bundles, and labeled medial olivocochlear (MOC) fibers crossing the tunnel of Corti (Figure 4 a). However, CGRP-immunoreactivity in type II afferents was only observed in young (prior to hearing onset) mice, possibly due to decreased expression through development or to re-localization of CGRP to the axonal terminals in the cochlear nucleus. This perhaps explains why previous studies found CGRP immunolabeling only in cochlear efferents (Cabanillas \& Luebke, 2002; Kitajiri et al., 1985; S. F. Maison et al., 2003; Safieddine \& Eybalin, 1992; Sliwinska-Kowalska et al., 1989). To test whether expression of Cgrpa in SGNs was specific to the CGRPa-EGFP(GENSAT) mouse, an additional mouse line was examined. The knock-in CGRPa-EGFP(Zylka) mouse line showed $\mathrm{GFP}^{+}$SGNs in the base of a one-week-old cochlea (Figure $4 \mathrm{~b}$ ). As in the CGRPa$E G F P(G E N S A T)$ mouse, these $\mathrm{GFP}^{+} \mathrm{SGNs}$ showed partial overlap with peripherin-positive SGNs (Figure $4 \mathrm{c}-\mathrm{e}$ ), confirming the type II identity in a subset of $\mathrm{GFP}^{+} \mathrm{SGNs}$. However, 
reporter expression was not as robust in this mouse line, and labeling was unconvincing in older cochleas, again suggesting that Cgrpa expression may decline with maturation.

\section{Cgrpa and Th showed opposing gene expression gradients in SGNs along the cochlear spiral}

The expression of $C g r p a$ along the cochlear spiral was quantified by counting labeled somata in the spiral ganglion of CGRPa-EGFP(GENSAT) mouse cochleas (Figure 5). Each cochlear whole mount was divided into 10 equal segments in ImageJ (Figure $5 \mathrm{~b}$ ) and cell counts were recorded per segment. As seen in the sample cochlea (P30; Figure $5 \mathrm{c}$ ) and in the averaged data (Figure $5 \mathrm{~d}$ ), $\mathrm{GFP}^{+}$SGNs were less common in apical segments of the cochlea. This rising gradient toward the cochlear base was present both before and after the onset of hearing, although the total number of $\mathrm{GFP}^{+} \mathrm{SGNs}$ was greater in the pre-hearing postnatal cochlea (Figure $5 \mathrm{~d}$ ). Variability in the amplitude of the gradient was also observed among individual cochleas.

Previous work has shown $\mathrm{TH}$ as a biomarker for type II afferent neurons, particularly in the cochlear apex (Vyas et al., 2016). To compare with Cgrpa cochlear expression patterns, the numbers of $\mathrm{TH}^{+} \mathrm{SGNs}$ along the cochlea were quantified using the same method. $\mathrm{TH}^{+}$ SGNs were labeled by TH antibody immunostaining or by heavy dose of tamoxifen injection to achieve maximal induction of reporter expression in $\mathrm{Th}^{2 A-C r e E R}$; $\mathrm{Ai3}$ or $T h^{2 A-C r e E R}$; $A i 9$ mice. Specifically, to label pre-hearing $T h^{+}$SGNs, multiple doses of tamoxifen were administrated through intragastric or intraperitoneal injections before P10; to label $\mathrm{Th}^{+}$SGNs in hearing mice, tamoxifen was administrated through gavage after P12.

In contrast to the expression pattern of $C_{g r p a}, \mathrm{TH}^{+} \mathrm{SGNs}$ were more numerous in apical segments (Figure 6). This declining gradient from apex to base was observed with both labeling strategies, though the mouse line usually labels fewer SGNs, possibly due to inadequate recombination (Figure $6 \mathrm{~b}$ ). There was little difference in the number of $\mathrm{TH}^{+}$ SGNs (quantified with TH immunolabeling) or the pattern of expression between cochleas before and after hearing onset (Figure $6 \mathrm{c}$ ). Furthermore, tamoxifen injection before hearing onset results in co-extensive labeling of SGNs with TH immunostaining performed after hearing onset (data not shown), suggesting Th is expressed in a stable set of SGNs before and after hearing onset.

For direct comparison, the average number of $\mathrm{TH}^{+}$SGNs (labeled by $\mathrm{TH}$ immunostaining) per bin was plotted together with that of the $\mathrm{GFP}^{+}$SGNs in CGRPa-EGFP(GENSAT) cochleas after hearing onset (Figure 7). The opposing gradients of these two distributions are evident, though the average SGN counts for Cgrpa-driven reporter expression are clearly larger than that of $\mathrm{TH}^{+}$SGNs. These two gradients show significant overlap in the middle portions of the cochlea.

To investigate whether the expression of Cgrpa and $T h$ are mutually exclusive in type II afferent neurons at the medial turn, $T h^{2 A-C r e E R}$; Ai9; CGRPa-EGFP(GENSAT) mouse cochleas were obtained and examined. A fraction of SGNs in mid-regions of the cochlea were positive for both GFP and TdTomato immunolabel (detected by an antibody against 
dsRed), but not for that against TuJ1 (marking type I SGNs), suggesting these are type II SGNs that co-express Cgrpa and Th (Figure 8).

\section{DISCUSSION}

In the present study, the gene Calca/Cgrpa is shown to be expressed in type II versus type I afferent neurons after hearing onset. Furthermore, Calca/Cgrpa drives reporters preferentially in 'higher frequency' type II SGNs nearer the cochlear base, producing a spatial expression gradient complementary to that of $T h$, which is expressed preferentially in apical ('lower frequency') type II SGNs.

\section{Mouse genetic tools to selectively target type II afferent fibers}

Since the earliest descriptions of cochlear innervation, the unusual innervation pattern of type II afferents has attracted attention and conjecture (Lorente De Nó, 1976). Early proposals took the logical view that the extensive connectivity of 'external spiral fibers' (spironeuren) with OHCs on the more-mobile portion of the basilar membrane implied greater acoustic sensitivity (Davis, 1961; von Békésy, 1960), or that they served as sensors to modulate the set-point of OHC activity, akin to the role of muscle spindle afferents (Kim, 1984). While functional studies remain hampered by the scarcity and small, unmyelinated processes of type II afferents, heroic efforts provided one anatomically-confirmed recording in vivo (Robertson, 1984), and characterization of several more identified by their much slower antidromic conduction velocity (Brown, 1994; Robertson et al., 1999). In contrast to those earlier expectations of greater acoustic sensitivity, none of these afferents had spontaneous activity, and all were in sensitive or unresponsive to sound (of 28 fibers reported in these publications, only 1 responded to broadband noise at $80 \mathrm{~dB}$ SPL). This acoustic insensitivity and morphological similarity to somatic C-fibers led to speculation that type II afferents may signal tissue trauma (Tonndorf, 1987) rather than sound per se, as has been supported by experimental findings in vivo (Flores et al., 2015) and ex vivo (C. Liu et al., 2015; C. Weisz, Glowatzki, \& Fuchs, 2009; C. J. Weisz et al., 2012).

Further exploration of type II function will benefit from genetic tools that enable selective manipulations for morphological, physiological and behavioral analyses. Type II afferents are preferentially labeled by antibodies to the intermediate filament protein peripherin (Hafidi, 1998; Huang, Thorne, Housley, \& Montgomery, 2007) prompting its use as a genetic tool, but with mixed results (Froud et al., 2015; S. Maison et al., 2016), possibly due to more widespread expression during development (Hafidi et al., 1993). Additional type IIspecific genes can be identified by gene-profiling strategies, or by survey of selective biomarkers found in analogous cell types, the strategy adopted here. Th and Cgrpa, two genes expressed in somatic C-fibers, were found to be expressed selectively in type II SGNs after hearing onset, suggesting their use as genetic tools. However, caution should be taken to avoid confounding effects due to the expression of these genes in other neuronal types that could influence cochlear function. Cgrpa is expressed in medial and lateral efferents (Cabanillas \& Luebke, 2002; Kitajiri et al., 1985; S. F. Maison et al., 2003; Safieddine \& Eybalin, 1992; Sliwinska-Kowalska et al., 1989). Th is also expressed in lateral efferents and sympathetic fibers (Darrow, Simons, Dodds, \& Liberman, 2006). Because the cell bodies of 
these other neuron types are located outside of the cochlea, a combination of mouse genetic tools with local viral injection may help to affect type II afferent fibers selectively. However, due to the graded expression of Th and Cgrpa in type II SGNs, genetic tools based on them would affect only a subset of type II SGNs. Recent work describes Gata3, a transcription factor that is expressed in all SGNs during development, that could be a type II marker in adult cochlea (Nishimura et al., 2017). However, it remains to be determined whether Gata3 labels the whole type II neuron population.

\section{Genes expressed in small diameter DRG neurons are found in type II afferent neurons}

Small diameter, unmyelinated $\mathrm{C}$ fibers encode a variety of noxious sensations and can be subdivided by molecular markers (Green \& Dong, 2016; Le Pichon \& Chesler, 2014). TH is expressed in C-fibers that normally convey innocuous mechanical sensations, but could also modulate pain, reviewed in (Brumovsky, 2016) and CGRP is a well-established biomarker for peptidergic pain-sensing C-fibers (Russell et al., 2014). In the present work, Calca/ Cgrpa was found to be expressed by type II but not type I afferent neurons after hearing onset. In the pre-hearing cochlea, CGRP immunolabeling was found in type II spiral processes, confirming the expression in type II SGNs. The expression of the marker for peptidergic C-fibers in type II afferents supports the hypothesis that these neurons might function as 'cochlear nociceptors' and carry noxious sensations arising subsequent to hearing loss. The preferential expression of Cgrpa promoter-driven reporters in basallylocated type II afferents adds further weight to this hypothesis. Hearing loss and cochlear damage progress from the high frequency cochlear base to the lower frequency cochlear apex. Type II afferents in the vulnerable cochlear base would be positioned to drive inflammation and other pro-nociceptive changes by the release of CGRP. Although several observations suggest developmental downregulation of CGRP expression in the SGNs, this does not invalidate this hypothesis. The synthesis of CGRP has shown to be upregulated by inflammatory pain (Nakanishi et al., 2010). Tissue damage or inflammation in the cochlea should be minimal at 3-week to 2-month-old, the age range used in the present study. CGRP expression may therefore be at a low basal level. Incidentally, the relatively higher expression of CGRP in SGNs of the one-week-old cochlea also could be induced by the extensive developmental processes occurring during the first postnatal week, including the pruning of type I afferent dendrites and loss of type II SGNs (Barclay et al., 2011). It remains unknown whether and where CGRP might be released from type II afferent fibers. Release from the peripheral dendrites could signal inflammation in the cochlea, while release from the axonal terminals in the brainstem could modulate the sensitivity of the postsynaptic ascending neurons. Furthermore, previous work described purinergic responses in type II afferent fibers (C. Liu et al., 2015), suggesting type II fibers respond to ATP as a signal of tissue damage. Interestingly, CGRP released by trigeminal nociceptive neurons has been shown to upregulate $\mathrm{P}_{2} \mathrm{X}_{3}$ expression in these neurons; a similar mechanism could be adopted by type II afferent fibers (Fabbretti et al., 2006). Finally, the preferential expression of TH in apical type II afferents suggests that dopamine may be released by type II afferent fibers. By analogy to the somatosensory system, dopamine may function to modulate the nociceptive signal. However, all the aforementioned roles of CGRP and TH in type II afferent fibers are speculative. Future experiments are needed to test these hypotheses. 


\section{Heterogeneity among type II afferent neurons}

Sensory systems such as vision and somatosensation utilize diverse types of primary afferent neurons, together with their different central projections, to encode different perceptual submodalities associated with the sensory inputs. For example, different types of DRG neurons with their different morphologies and molecular characterizations are specialized to sense touch, temperature, pain, proprioception, etc. Cochlear afferents carry information to the cochlear nucleus where the perceived sound features, including pitch, loudness, timbre, duration and location, begin to be directed to separate central circuits. Given their relative insensitivity to sound, type II afferent neurons are unlikely to be involved in this processing. Rather, damaging levels of sound increase activity-dependent c-Fos labeling in the granule cell domain of the cochlear nucleus complex (Flores et al., 2015), the locus of type II synaptic contacts (Benson \& Brown, 2004; Brown \& Ledwith, 1990). Given the differential expression patterns of Th and Cgrpa reported here, it will be of interest to determine the central projections of these differentiated type II afferent neurons.

The present work describes a spatial gene expression gradient of Th and Cgrpa in type II SGNs. It is unclear whether these gradients are stable and represent subpopulations of type II afferent neurons, or flexible and subject to change depending on the sound environment. For Cgrpa, the amplitude of its expression gradient decreases after hearing onset and varies among different cochleas, suggesting the possibility of plasticity. In view of the proposed nociceptive role of type II afferent neurons, future experiments are required to ask if the population of $\mathrm{Th}^{+}$and $\mathrm{Cgrpa}^{+}$type II afferent neurons could change upon acoustic damage. The type II marker peripherin also may be heterogeneous. Peripherin expression appears to be down-regulated during development in a subset of Gata3 ${ }^{+}$type II SGNs (Nishimura et al., 2017). Previous work has shown that only a subset of TH-positive type II afferent neurons were peripherin-positive (Vyas et al., 2016). Maison Liberman \& Liberman (2016) also noted that peripherin robustly labeled type II SGN somata, but only weakly and infrequently labeled projections in the adult mice. However, the differential expression of peripherin does not seem to present a tonotopic gradient, suggesting another level of diversity in the genetic profile of type II neurons. It is likely that a combination of many genes, rather than the expression level of a single or several marker genes defines the functional status of each subset of type II neurons as a function of cochlear position, age and experience.

\section{Acknowledgments}

We thank Drs. David Ginty and Amanda Zimmerman (Harvard University, MA) for the CGRPa-EGFP(GENSAT) and $T h^{2 A-C r e E R}$ mice, Drs. Mark Zylka and Eric McCoy (University of North Carolina, NC) for sharing cochlear tissues of CGRPa-EGFP (Zylka) mouse, Dr. Jay Pasricha (Johns Hopkins Hospital, MD) for the CGRPaCreER mouse, Fatima Chakir for genotyping and Hamad Javaid for help with SGN quantification.

Supported by NIDCD R01DC006476 and R01DC012957 to EG, NIDCD R01DC011741 and R01DC016559 to PAF, NIDCD P30 DC005211 to the Center for Hearing and Balance, the John Mitchell, Jr. Trust, the David M. Rubenstein Fund for Hearing Research and the John E. Bordley Professorship (PAF).

\section{References}

Abraira VE, Ginty DD. The sensory neurons of touch. Neuron. 2013; 79(4):618-639. DOI: 10.1016/ j.neuron.2013.07.051 [PubMed: 23972592] 
Abraira VE, Kuehn ED, Chirila AM, Springel MW, Toliver AA, Zimmerman AL, Ginty DD. The Cellular and Synaptic Architecture of the Mechanosensory Dorsal Horn. Cell. 2017; 168(1-2):295310 e219. DOI: 10.1016/j.cell.2016.12.010 [PubMed: 28041852]

Assas BM, Pennock JI, Miyan JA. Calcitonin gene-related peptide is a key neurotransmitter in the neuro-immune axis. Front Neurosci. 2014; 8:23.doi: 10.3389/fnins.2014.00023 [PubMed: 24592205]

Bai L, Lehnert BP, Liu J, Neubarth NL, Dickendesher TL, Nwe PH, Ginty DD. Genetic Identification of an Expansive Mechanoreceptor Sensitive to Skin Stroking. Cell. 2015; 163(7):1783-1795. DOI: 10.1016/j.cell.2015.11.060 [PubMed: 26687362]

Barclay M, Ryan AF, Housley GD. Type I vs type II spiral ganglion neurons exhibit differential survival and neuritogenesis during cochlear development. Neural Dev. 2011; 6:33.doi: 10.1186/1749-8104-6-33 [PubMed: 21989106]

Basbaum AI, Bautista DM, Scherrer G, Julius D. Cellular and molecular mechanisms of pain. Cell. 2009; 139(2):267-284. DOI: 10.1016/j.cell.2009.09.028 [PubMed: 19837031]

Benson TE, Brown MC. Postsynaptic targets of type II auditory nerve fibers in the cochlear nucleus. J Assoc Res Otolaryngol. 2004; 5(2):111-125. DOI: 10.1007/s10162-003-4012-3 [PubMed: 15357415]

Brown MC. Antidromic responses of single units from the spiral ganglion. J Neurophysiol. 1994; 71(5):1835-1847. [PubMed: 8064351]

Brown MC, Ledwith JV 3rd. Projections of thin (type-II) and thick (type-I) auditory-nerve fibers into the cochlear nucleus of the mouse. Hear Res. 1990; 49(1-3):105-118. [PubMed: 1963423]

Brumovsky PR. Dorsal root ganglion neurons and tyrosine hydroxylase-an intriguing association with implications for sensation and pain. Pain. 2016; 157(2):314-320. DOI: 10.1097/j.pain. 0000000000000381 [PubMed: 26447702]

Cabanillas LA, Luebke AE. CGRP- and cholinergic-containing fibers project to guinea pig outer hair cells. Hear Res. 2002; 172(1-2):14-17. [PubMed: 12361863]

Chee MJ, Pissios P, Maratos-Flier E. Neurochemical characterization of neurons expressing melaninconcentrating hormone receptor 1 in the mouse hypothalamus. J Comp Neurol. 2013; 521(10): 2208-2234. DOI: 10.1002/cne.23273 [PubMed: 23605441]

Darrow KN, Simons EJ, Dodds L, Liberman MC. Dopaminergic innervation of the mouse inner ear: evidence for a separate cytochemical group of cochlear efferent fibers. J Comp Neurol. 2006; 498(3):403-414. DOI: 10.1002/cne.21050 [PubMed: 16871528]

Davies D. Temporal and spatial regulation of alpha6 integrin expression during the development of the cochlear-vestibular ganglion. J Comp Neurol. 2007; 502(5):673-682. DOI: 10.1002/cne.21302 [PubMed: 17436285]

Davis H. Some principles of sensory receptor action. Physiol Rev. 1961; 41:391-416. [PubMed: 13720173]

Du Y, Ma Z, Lin S, Dodel RC, Gao F, Bales KR, Paul SM. Minocycline prevents nigrostriatal dopaminergic neurodegeneration in the MPTP model of Parkinson's disease. Proc Natl Acad Sci U S A. 2001; 98(25):14669-14674. DOI: 10.1073/pnas.251341998 [PubMed: 11724929]

Echteler SM, Nofsinger YC. Development of ganglion cell topography in the postnatal cochlea. J Comp Neurol. 2000; 425(3):436-446. [PubMed: 10972943]

Fabbretti E, D'Arco M, Fabbro A, Simonetti M, Nistri A, Giniatullin R. Delayed upregulation of ATP $\mathrm{P} 2 \mathrm{X} 3$ receptors of trigeminal sensory neurons by calcitonin gene-related peptide. J Neurosci. 2006; 26(23):6163-6171. DOI: 10.1523/JNEUROSCI.0647-06.2006 [PubMed: 16763024]

Flores-Otero J, Davis RL. Synaptic proteins are tonotopically graded in postnatal and adult type I and type II spiral ganglion neurons. J Comp Neurol. 2011; 519(8):1455-1475. DOI: 10.1002/cne. 22576 [PubMed: 21452215]

Flores EN, Duggan A, Madathany T, Hogan AK, Marquez FG, Kumar G, Garcia-Anoveros J. A noncanonical pathway from cochlea to brain signals tissue-damaging noise. Curr Biol. 2015; 25(5): 606-612. DOI: 10.1016/j.cub.2015.01.009 [PubMed: 25639244]

Froud KE, Wong AC, Cederholm JM, Klugmann M, Sandow SL, Julien JP, Housley GD. Type II spiral ganglion afferent neurons drive medial olivocochlear reflex suppression of the cochlear amplifier. Nat Commun. 2015; 6:7115.doi: 10.1038/ncomms8115 [PubMed: 25965946] 
Gong S, Zheng C, Doughty ML, Losos K, Didkovsky N, Schambra UB, Heintz N. A gene expression atlas of the central nervous system based on bacterial artificial chromosomes. Nature. 2003; 425(6961):917-925. DOI: 10.1038/nature02033 [PubMed: 14586460]

Green D, Dong X. The cell biology of acute itch. J Cell Biol. 2016; 213(2):155-161. DOI: 10.1083/ jcb.201603042 [PubMed: 27114499]

Hafidi A. Peripherin-like immunoreactivity in type II spiral ganglion cell body and projections. Brain Res. 1998; 805(1-2):181-190. [PubMed: 9733963]

Hafidi A, Despres G, Romand R. Ontogenesis of type II spiral ganglion neurons during development: peripherin immunohistochemistry. Int J Dev Neurosci. 1993; 11(4):507-512. [PubMed: 8237466]

Hayes L, Zhang Z, Albert P, Zervas M, Ahn S. Timing of Sonic hedgehog and Gli1 expression segregates midbrain dopamine neurons. J Comp Neurol. 2011; 519(15):3001-3018. DOI: 10.1002/ cne.22711 [PubMed: 21713771]

Hegarty DM, Tonsfeldt K, Hermes SM, Helfand H, Aicher SA. Differential localization of vesicular glutamate transporters and peptides in corneal afferents to trigeminal nucleus caudalis. J Comp Neurol. 2010; 518(17):3557-3569. DOI: 10.1002/cne.22414 [PubMed: 20593358]

Huang LC, Thorne PR, Housley GD, Montgomery JM. Spatiotemporal definition of neurite outgrowth, refinement and retraction in the developing mouse cochlea. Development. 2007; 134(16):29252933. DOI: 10.1242/dev.001925 [PubMed: 17626062]

Ivanova E, Lee P, Pan ZH. Characterization of multiple bistratified retinal ganglion cells in a purkinje cell protein 2-Cre transgenic mouse line. J Comp Neurol. 2013; 521(9):2165-2180. DOI: 10.1002/ cne.23279 [PubMed: 23224947]

Kim, DO. Functional roles of inner- and outer-hair sub-system in the cochlea and brainstem. In: Berlin, CI., editor. Hearing Science: Recent Advances. San Diego CA: College Press; 1984. p. 241-262.

Kitajiri M, Yamashita T, Tohyama Y, Kumazawa T, Takeda N, Kawasaki Y, et al. Localization of calcitonin gene-related peptide in the organ of Corti of the rat: an immunohistochemical study. Brain Res. 1985; 358(1-2):394-397. [PubMed: 3907750]

Kong JH, Adelman JP, Fuchs PA. Expression of the SK2 calcium-activated potassium channel is required for cholinergic function in mouse cochlear hair cells. Journal of Physiology-London. 2008; 586(22):5471-5485. DOI: 10.1113/jphysiol.2008.160077

Korrapati S, Roux I, Glowatzki E, Doetzlhofer A. Notch signaling limits supporting cell plasticity in the hair cell-damaged early postnatal murine cochlea. Plos One. 2013; 8(8):e73276.doi: 10.1371/ journal.pone.0073276 [PubMed: 24023676]

Lallemend F, Vandenbosch R, Hadjab S, Bodson M, Breuskin I, Moonen G, Malgrange B. New insights into peripherin expression in cochlear neurons. Neuroscience. 2007; 150(1):212-222. DOI: 10.1016/j.neuroscience.2007.08.032 [PubMed: 17964735]

Lang H, Li M, Kilpatrick LA, Zhu J, Samuvel DJ, Krug EL, Goddard JC. Sox2 up-regulation and glial cell proliferation following degeneration of spiral ganglion neurons in the adult mouse inner ear. $\mathrm{J}$ Assoc Res Otolaryngol. 2011; 12(2):151-171. DOI: 10.1007/s10162-010-0244-1 [PubMed: 21061038]

Le Pichon CE, Chesler AT. The functional and anatomical dissection of somatosensory subpopulations using mouse genetics. Front Neuroanat. 2014; 8:21.doi: 10.3389/fnana.2014.00021 [PubMed: 24795573]

Li L, Ginty DD. The structure and organization of lanceolate mechanosensory complexes at mouse hair follicles. Elife. 2014; 3:e01901.doi: 10.7554/eLife.01901 [PubMed: 24569481]

Liu C, Glowatzki E, Fuchs PA. Unmyelinated type II afferent neurons report cochlear damage. Proc Natl Acad Sci U S A. 2015; doi: 10.1073/pnas.1515228112

Liu W, Bostrom M, Rask-Andersen H. Expression of peripherin in the pig spiral ganglion - aspects of nerve injury and regeneration. Acta Oto-Laryngologica. 2009; 129(6):608-614. Pii 902089338. DOI: 10.1080/00016480802369294 [PubMed: 18763177]

Liu W, Kinnefors A, Bostrom M, Rask-Andersen H. Expression of peripherin in human cochlea. Cell Tissue Res. 2010; 342(3):345-351. DOI: 10.1007/s00441-010-1081-6 [PubMed: 21088854]

Lorente De Nó R. Some unresolved problems concerning the cochlear nerve. Ann Otol Rhinol Laryngol. 1976; 85(6 suppl 34 pt. 3):1-28. DOI: 10.1177/00034894760856S201 
Maison S, Liberman LD, Liberman MC. Type II Cochlear Ganglion Neurons Do Not Drive the Olivocochlear Reflex: Re-Examination of the Cochlear Phenotype in Peripherin Knock-Out Mice. eNeuro. 2016; 3(4)doi: 10.1523/ENEURO.0207-16.2016

Maison SF, Adams JC, Liberman MC. Olivocochlear innervation in the mouse: immunocytochemical maps, crossed versus uncrossed contributions, and transmitter colocalization. J Comp Neurol. 2003; 455(3):406-416. [PubMed: 12483691]

Maison SF, Liu XP, Vetter DE, Eatock RA, Nathanson NM, Wess J, Liberman MC. Muscarinic signaling in the cochlea: presynaptic and postsynaptic effects on efferent feedback and afferent excitability. J Neurosci. 2010; 30(19):6751-6762. DOI: 10.1523/JNEUROSCI.5080-09.2010 [PubMed: 20463237]

Martinez-Monedero R, Liu C, Weisz C, Vyas P, Fuchs PA, Glowatzki E. GluA2-Containing AMPA Receptors Distinguish Ribbon-Associated from Ribbonless Afferent Contacts on Rat Cochlear Hair Cells. eNeuro. 2016; 3(2)doi: 10.1523/ENEURO.0078-16.2016

McCoy ES, Taylor-Blake B, Zylka MJ. CGRP alpha-Expressing Sensory Neurons Respond to Stimuli that Evoke Sensations of Pain and Itch. Plos One. 2012; 7(5) doi:ARTN e36355 10.1371/ journal.pone.0036355.

McLean WJ, Smith KA, Glowatzki E, Pyott SJ. Distribution of the Na,K-ATPase alpha subunit in the rat spiral ganglion and organ of corti. J Assoc Res Otolaryngol. 2009; 10(1):37-49. DOI: 10.1007/ s10162-008-0152-9 [PubMed: 19082858]

Nakanishi M, Hata K, Nagayama T, Sakurai T, Nishisho T, Wakabayashi H, Yoneda T. Acid activation of Trpv1 leads to an up-regulation of calcitonin gene-related peptide expression in dorsal root ganglion neurons via the CaMK-CREB cascade: a potential mechanism of inflammatory pain. Mol Biol Cell. 2010; 21(15):2568-2577. DOI: 10.1091/mbc.E10-01-0049 [PubMed: 20534813]

Nishimura K, Noda T, Dabdoub A. Dynamic Expression of Sox2, Gata3, and Prox1 during Primary Auditory Neuron Development in the Mammalian Cochlea. Plos One. 2017; 12(1):e0170568.doi: 10.1371/journal.pone.0170568 [PubMed: 28118374]

Robertson D. Horseradish peroxidase injection of physiologically characterized afferent and efferent neurones in the guinea pig spiral ganglion. Hear Res. 1984; 15(2):113-121. [PubMed: 6490538]

Robertson D, Sellick PM, Patuzzi R. The continuing search for outer hair cell afferents in the guinea pig spiral ganglion. Hear Res. 1999; 136(1-2):151-158. [PubMed: 10511634]

Roux I, Hosie S, Johnson SL, Bahloul A, Cayet N, Nouaille S, Safieddine S. Myosin VI is required for the proper maturation and function of inner hair cell ribbon synapses. Human Molecular Genetics. 2009; 18(23):4615-4628. DOI: 10.1093/hmg/ddp429 [PubMed: 19744958]

Rueda J, de la Sen C, Juiz JM, Merchan JA. Neuronal loss in the spiral ganglion of young rats. Acta Otolaryngol. 1987; 104(5-6):417-421. [PubMed: 3434262]

Russell FA, King R, Smillie SJ, Kodji X, Brain SD. Calcitonin gene-related peptide: physiology and pathophysiology. Physiol Rev. 2014; 94(4):1099-1142. DOI: 10.1152/physrev.00034.2013 [PubMed: 25287861]

Safieddine S, Eybalin M. Triple Immunofluorescence Evidence for the Coexistence of Acetylcholine, Enkephalins and Calcitonin Gene-related Peptide Within Efferent (Olivocochlear) Neurons of Rats and Guinea-pigs. Eur J Neurosci. 1992; 4(10):981-992. [PubMed: 12106433]

Sanes JR, Masland RH. The types of retinal ganglion cells: current status and implications for neuronal classification. Annu Rev Neurosci. 2015; 38:221-246. DOI: 10.1146/annurevneuro-071714-034120 [PubMed: 25897874]

Sliwinska-Kowalska M, Parakkal M, Schneider ME, Fex J. CGRP-like immunoreactivity in the guinea pig organ of Corti: a light and electron microscopy study. Hear Res. 1989; 42(1):83-95. [PubMed: 2584160]

Song H, Yao E, Lin C, Gacayan R, Chen MH, Chuang PT. Functional characterization of pulmonary neuroendocrine cells in lung development, injury, and tumorigenesis. Proc Natl Acad Sci U S A. 2012; 109(43):17531-17536. DOI: 10.1073/pnas.1207238109 [PubMed: 23047698]

Tonndorf J. The analogy between tinnitus and pain: a suggestion for a physiological basis of chronic tinnitus. Hear Res. 1987; 28(2-3):271-275. [PubMed: 2820913]

von Békésy, G. Experiments in Hearing. New York: McGraw-Hill; 1960. 
Vyas P, Wu JS, Zimmerman A, Fuchs P, Glowatzki E. Tyrosine Hydroxylase Expression in Type II Cochlear Afferents in Mice. J Assoc Res Otolaryngol. 2016; doi: 10.1007/s10162-016-0591-7

Weisz C, Glowatzki E, Fuchs P. The postsynaptic function of type II cochlear afferents. Nature. 2009; 461(7267):1126-1129. DOI: 10.1038/nature08487 [PubMed: 19847265]

Weisz CJ, Lehar M, Hiel H, Glowatzki E, Fuchs PA. Synaptic transfer from outer hair cells to type II afferent fibers in the rat cochlea. J Neurosci. 2012; 32(28):9528-9536. DOI: 10.1523/ JNEUROSCI.6194-11.2012 [PubMed: 22787038]

Xing Y, Samuvel DJ, Stevens SM, Dubno JR, Schulte BA, Lang H. Age-related changes of myelin basic protein in mouse and human auditory nerve. Plos One. 2012; 7(4):e34500.doi: 10.1371/ journal.pone.0034500 [PubMed: 22496821]

Young ED. Neural representation of spectral and temporal information in speech. Philos Trans R Soc Lond B Biol Sci. 2008; 363(1493):923-945. DOI: 10.1098/rstb.2007.2151 [PubMed: 17827107] 

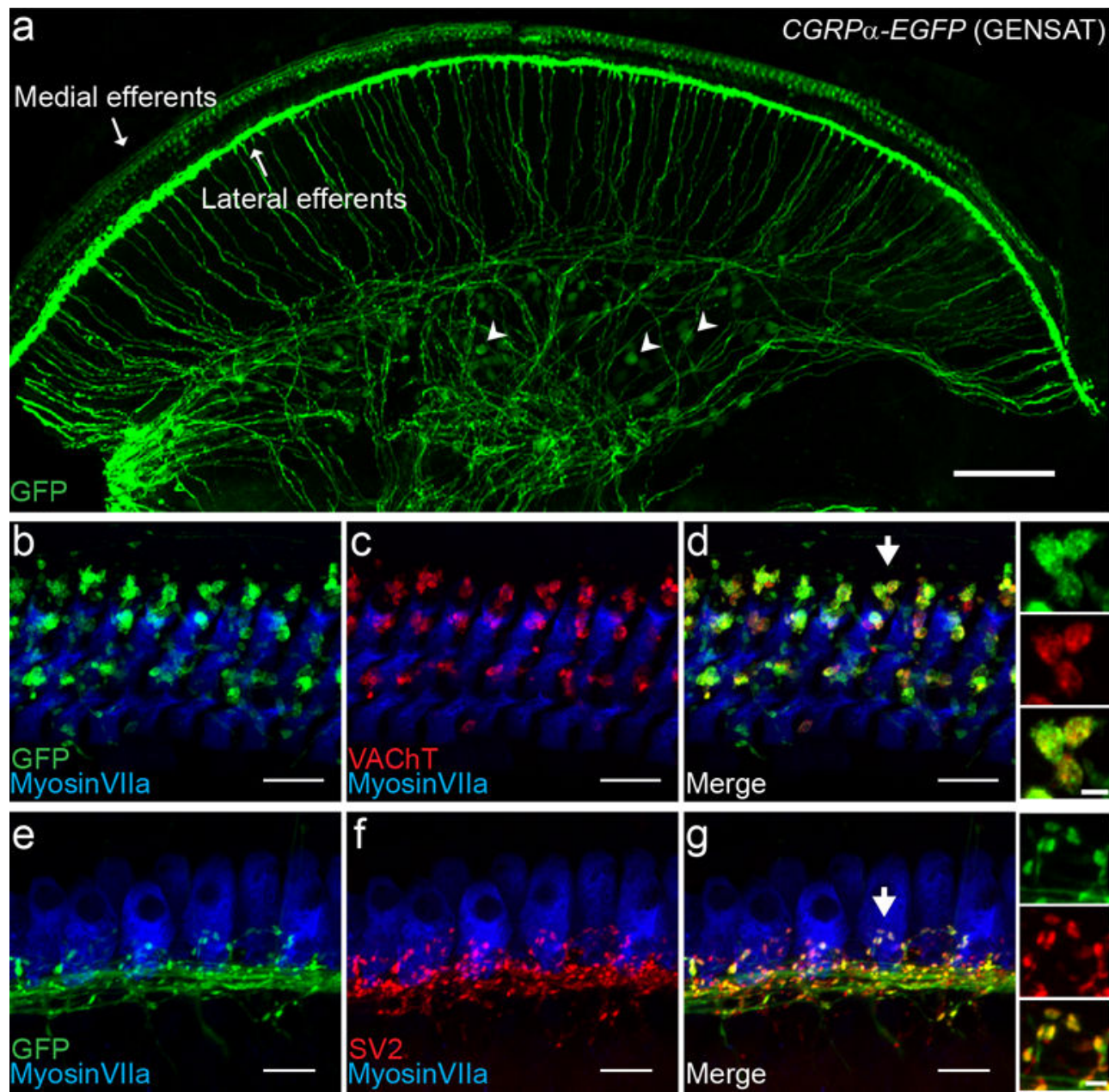

Figure 1. Young adult CGRPa-EGFP (GENSAT) cochlea labels medial efferents, lateral efferents and some SGNs

A basal piece of a one-month-old CGRPa-EGFP(GENSAT) mouse cochlea demonstrating the labeling of medial efferents, lateral efferents and some SGNs (a few indicated with arrowheads) (a). In a more zoomed in view (b), the endings on the OHCs labeled in the $C G R P a-E G F P$ (GENSAT) cochlea belong to medial efferents, as confirmed by co-labeling with VAChT (vesicular acetylcholine transporter) (c-d). Similarly, the co-labeling with SV2 (synaptic vesicle protein 2) confirms that the endings under the IHCs belong to the lateral efferents (e-g). Insets to the right of $\mathbf{d}$ and $\mathbf{g}$ : an enlarged view of the medial and lateral efferent endings, marked by the arrows in $\mathbf{d}$ and $\mathbf{g}$, respectively, for better visualization of the overlapping staining. Scale bar: $100 \mu \mathrm{m}$ in $\mathbf{a}, 10 \mu \mathrm{m}$ in b-g, and $2 \mu \mathrm{m}$ in the Insets. 

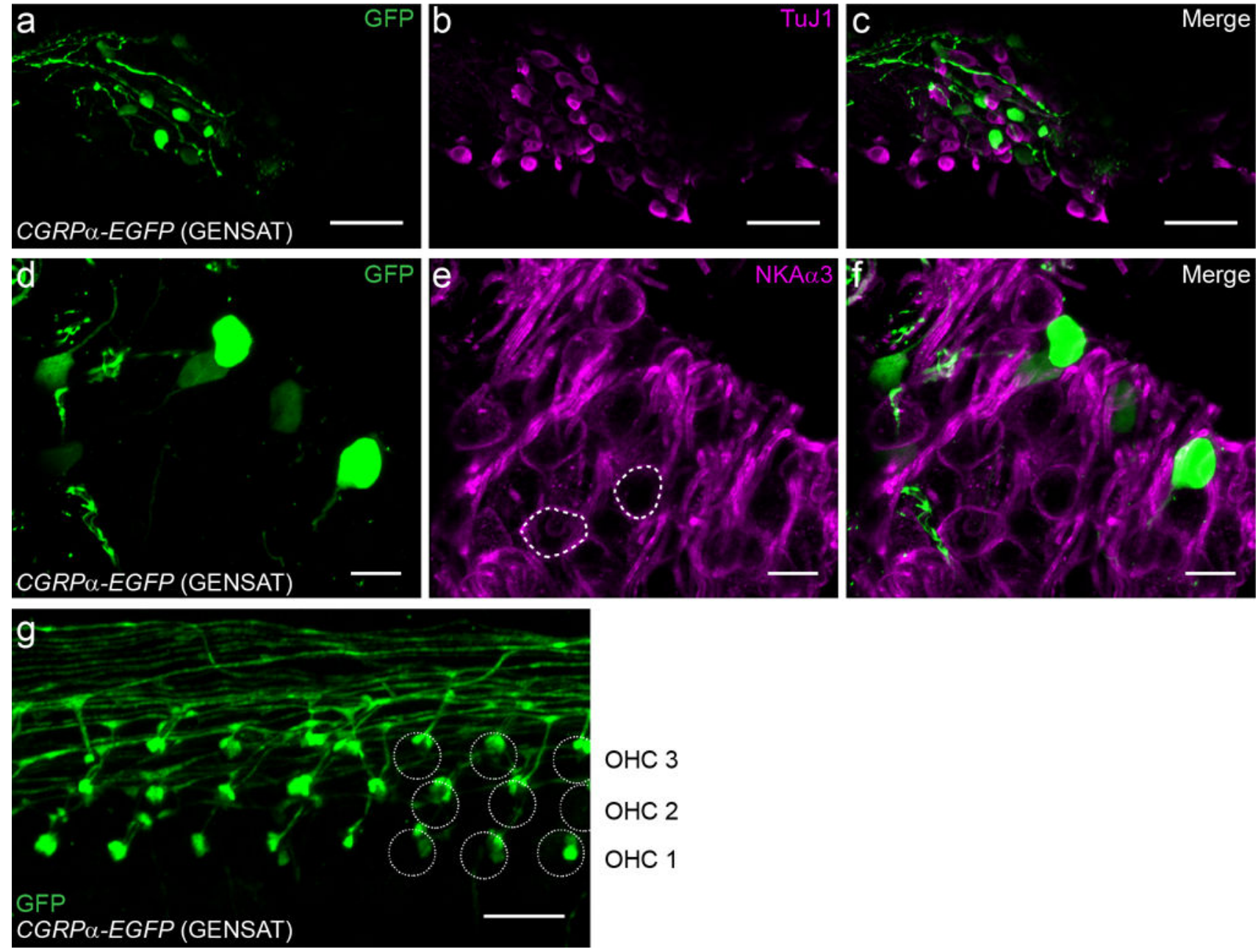

Figure 2. Young adult CGRP $a$-EGFP (GENSAT) cochlea labels type II SGNs

The non-overlapping labeling with type I SGN markers TuJ1 ( $\beta 3$ tubulin) (a-c) and NKAa3 (a $3 \mathrm{Na}^{+} / \mathrm{K}^{+}$ATPase) (e-f) suggests that the young adult CGRPa-EGFP(GENSAT) mouse cochlea labels type II SGNs. In e, two type I SGNs were circled to help visualize the plasma membrane labeling by NKAa3 antibody. A selective region of OHCs demonstrates the typical type II fiber endings labeled in CGRPa-EGFP(GENSAT) mouse cochlea (g). Mouse age: 3-4 week old for a-c, P30 for e-g. Scale bar: $50 \mu \mathrm{m}$ in a-c, and $10 \mu \mathrm{m}$ in e-g. 

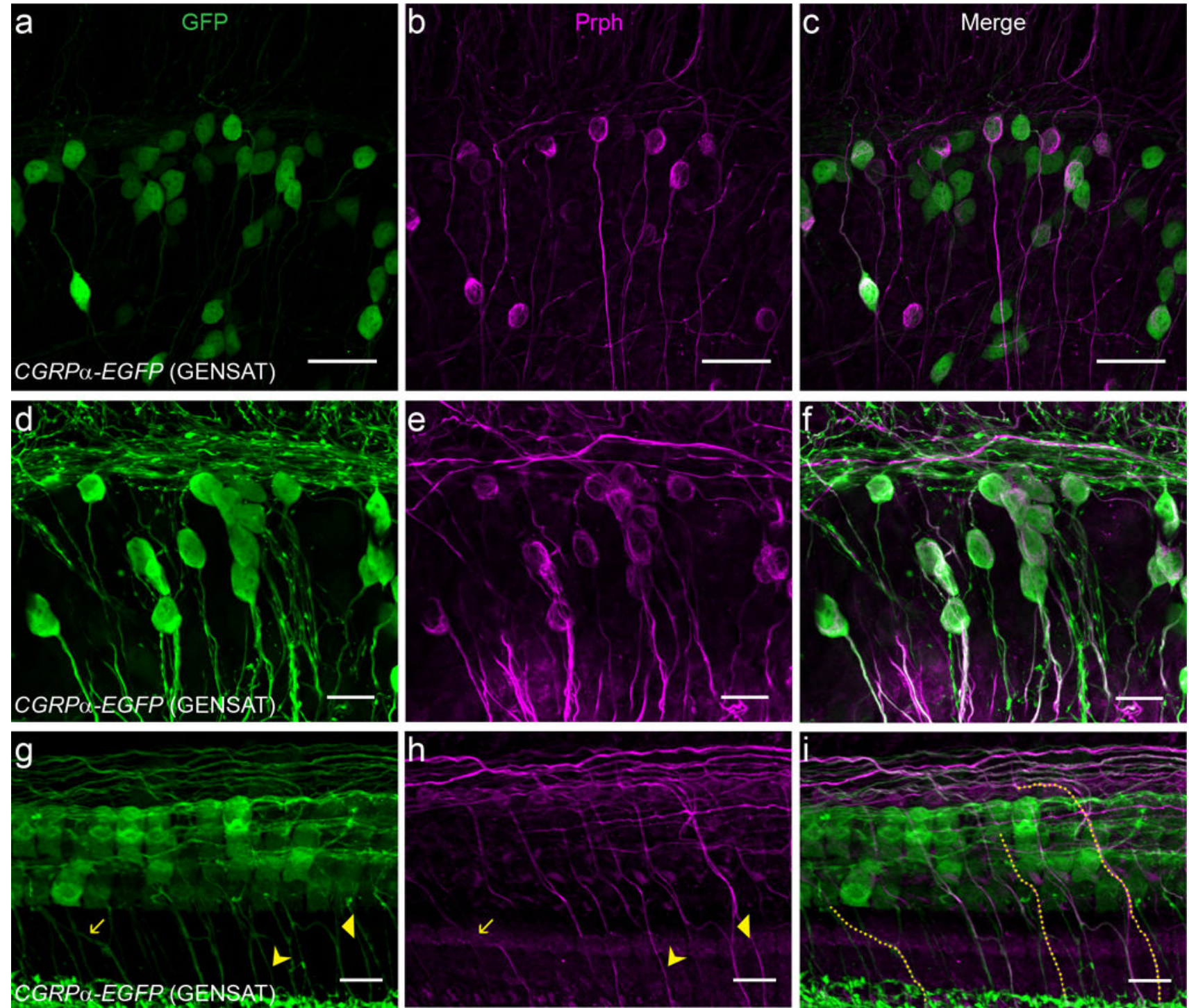

Figure 3. One-week-old CGRPa-EGFP (GENSAT) cochleas show partially overlapping labeling of SGNs with peripherin

One-week-old CGRPa-EGFP(GENSAT) mouse cochleas showed partially overlapping labeling with Prph (peripherin) in the SGN region (a-f) and hair cell region (g-i). For different cochlear preparation, the percentage of SGNs co-labeled by the mouse line and the Prph antibody varied from low (a-c) to high (d-f). Fibers co-labeled by the mouse line and the Prph antibody traveled across the tunnel of Corti (three such fibers are highlighted in $\mathbf{i}$, which are also indicated with arrows in $\mathbf{g}$ and $\mathbf{h}$ ), suggesting that they are type II fibers. Note also the bundles of type II fibers traveling near the outskirts of the OHC region that are labeled by both the mouse line and the Prph antibody. Scale bar: $40 \mu \mathrm{m}$ in a-c, $20 \mu \mathrm{m}$ in d-f, and $10 \mu \mathrm{m}$ in $\mathbf{g - i}$. 

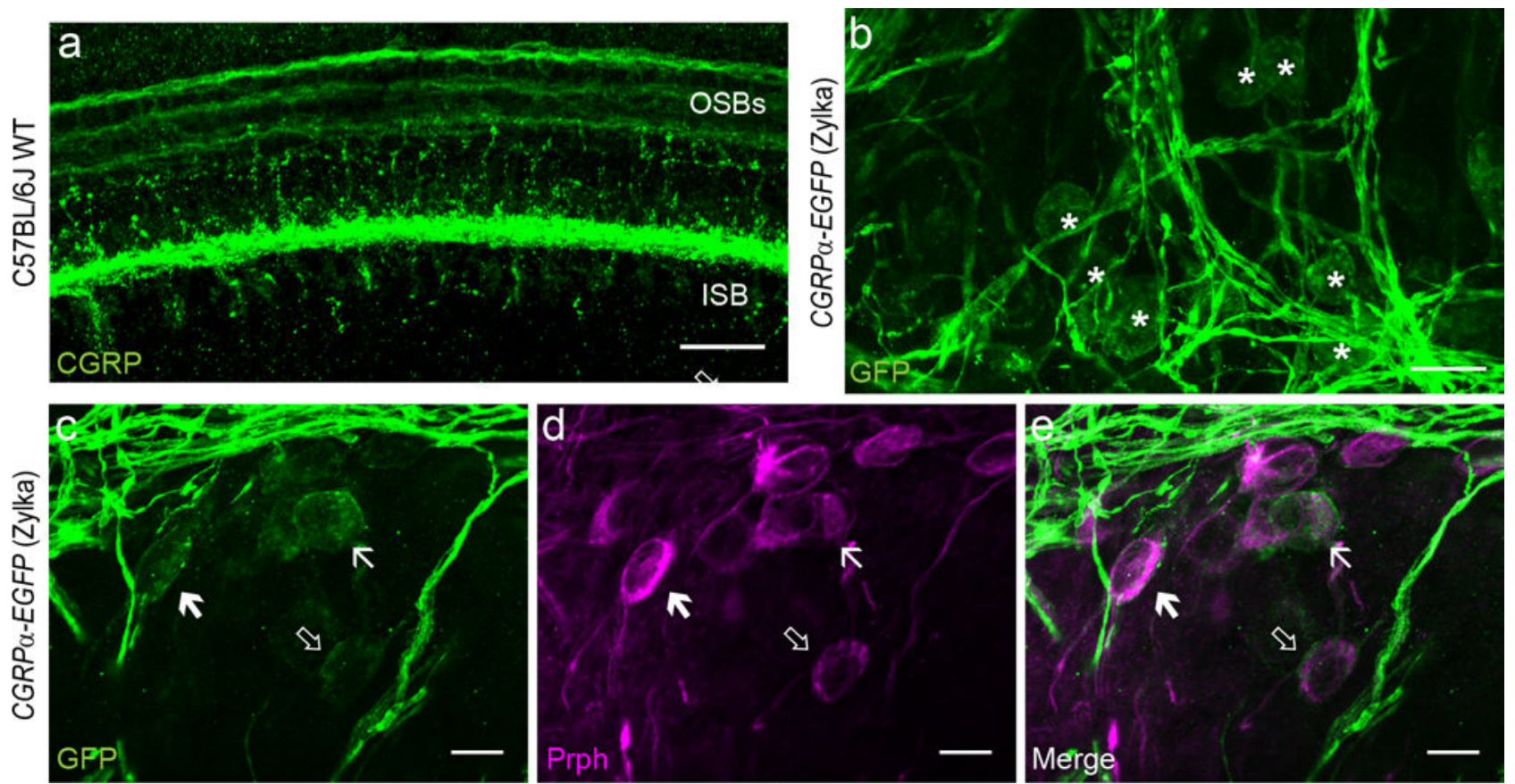

Figure 4. Cgrpa is expressed in type II afferent neurons of one-week-old mice CGRP antibody stained the three OSBs (outer spiral bundles) in the OHC region in P6 C57BL/6J WT (wild-type) mouse cochlea (a), suggesting that the CGRP peptide is expressed in type II fibers. A knock-in CGRPa-EGFP(Zylka) mouse line also showed labeling of SGNs in the base at P6-8 age (asterisks in b). These SGNs also showed partially overlapping labeling with Prph (arrows in c-e). Scale bar: $20 \mu \mathrm{m}$ in $\mathbf{a}-\mathbf{b}$, and $10 \mu \mathrm{m}$ in c-e. 


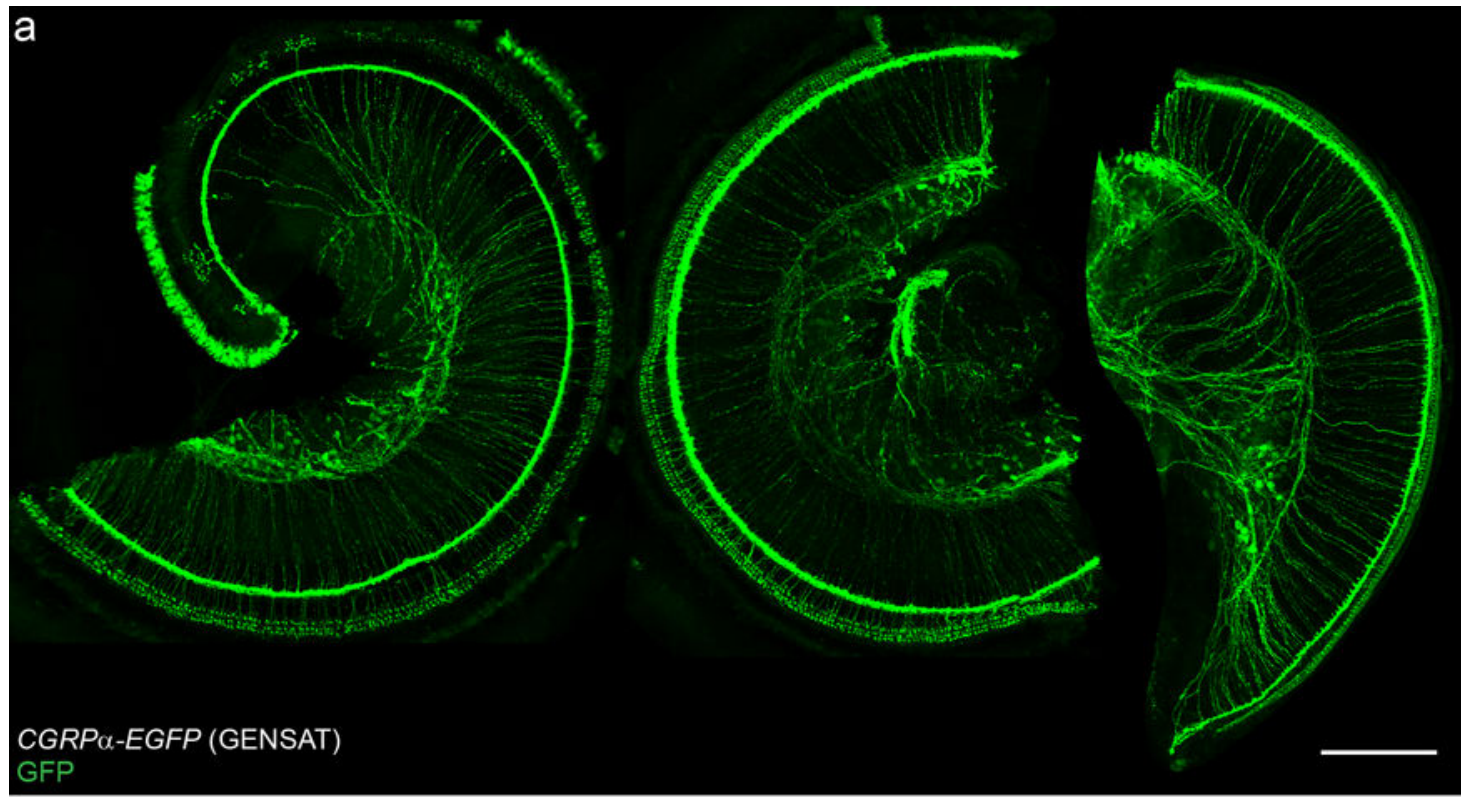

b
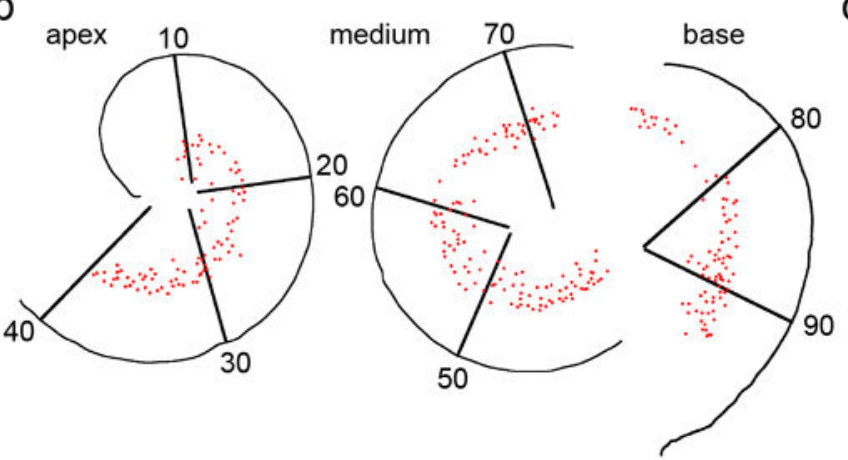

C

d
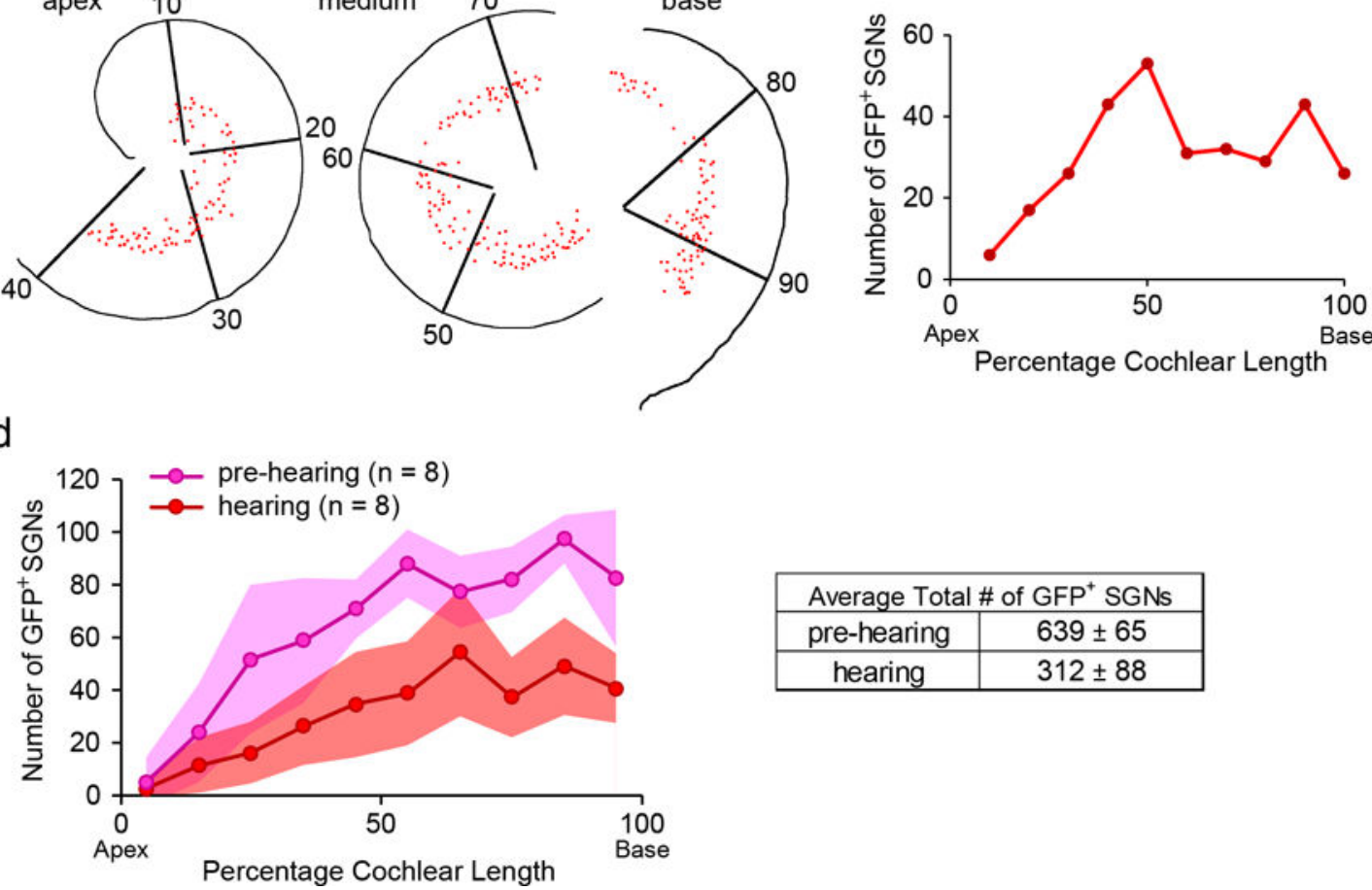

Figure 5. CGRPa-EGFP (GENSAT) cochlea shows a basal-to-apical gradient labeling of SGNs A representative cochlear whole mount of a one-month-old CGRPa-EGFP(GENSAT) mouse (a). Scale bar: $200 \mu \mathrm{m}$. The cochlear spiral shown in $\mathbf{a}$ is reconstructed in $\mathbf{b}$ with each red dot representing a $\mathrm{GFP}^{+} \mathrm{SGN}$. The $\mathrm{SGN}$ region is divided into 10 bins of equal length of cochlear spiral. The plot of the number of GFP ${ }^{+}$SGNs identified in each bin for this example is shown in c. Quantification of the number of $\mathrm{GFP}^{+} \mathrm{SGNs}_{\text {before }}(\mathrm{P} 6-8, \mathrm{n}=8)$ and after hearing onset $(\sim \mathrm{P} 30, \mathrm{n}=8)$ in $C G R P a-E G F P($ GENSAT) cochleas $(\mathbf{d})$. Shaded areas represent standard deviations. 


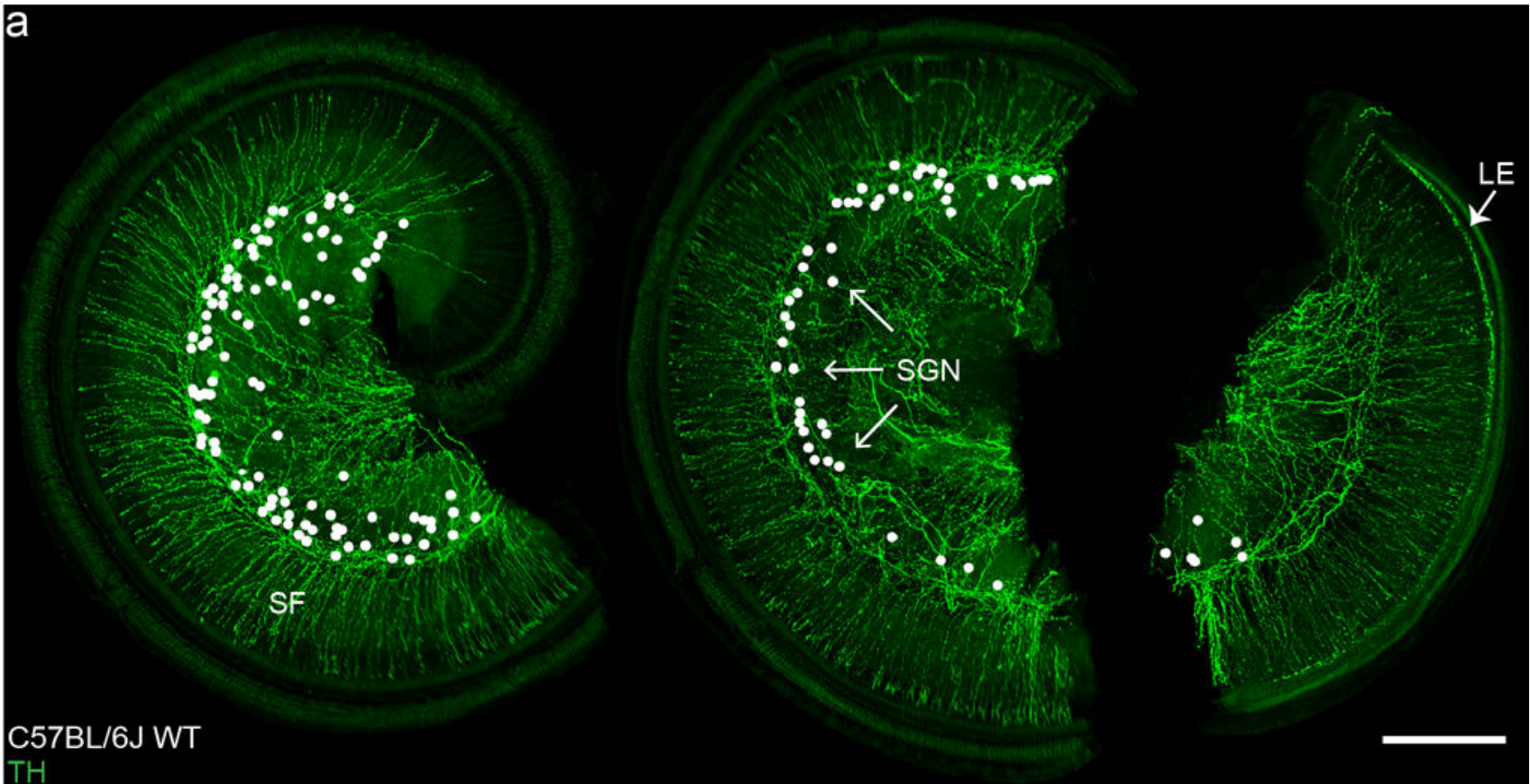

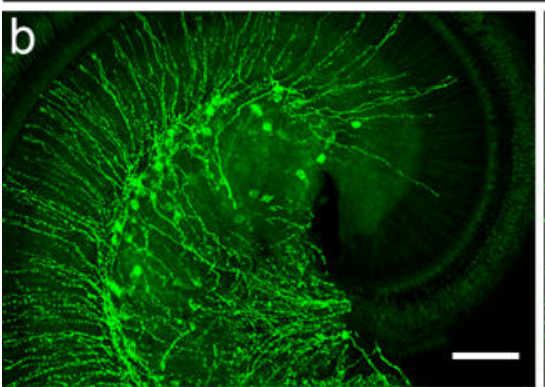

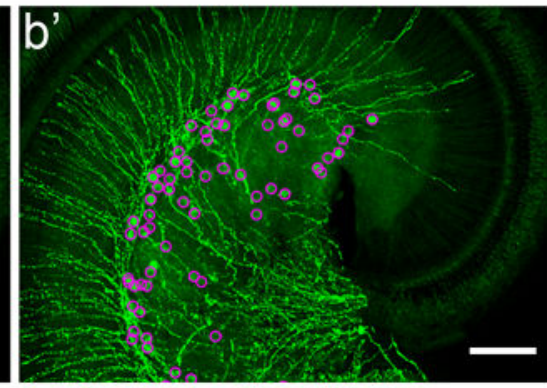

d

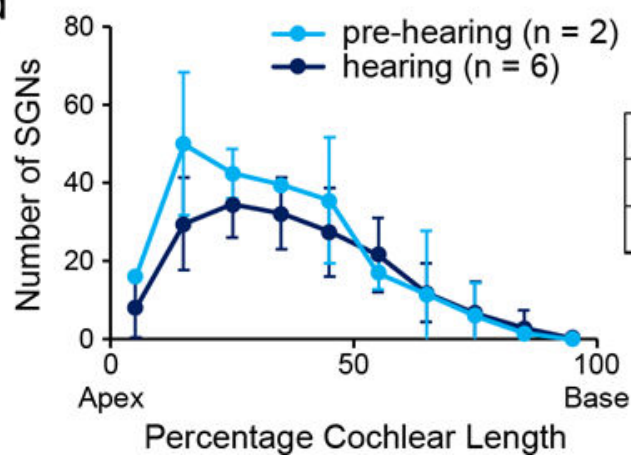

C

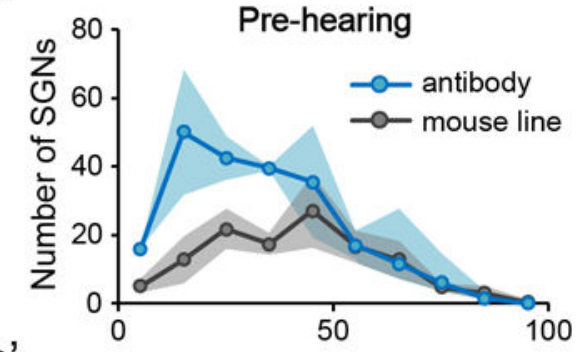

$C^{\prime}$

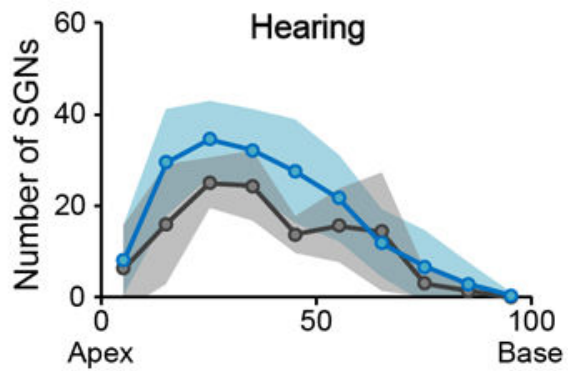

Percentage Cochlear Length

Figure 6. Th is expressed in type II afferent neurons with an apical-to-basal gradient

A representative cochlear whole mount of a two-month-old C57BL/6J WT mouse cochlea stained with TH antibody (a). TH antibody labeled SGNs in Rosenthal's canal (arrows), sympathetic fibers (SF) in the osseous spiral lamina, and lateral efferent (LE) fibers in the IHC area in the basal third of this cochlea (arrow). White dots indicate the locations of each identified $\mathrm{TH}^{+} \mathrm{SGN}$ in this cochlea. To demonstrate how the $\mathrm{TH}^{+} \mathrm{SGN}$ s were identified. The original image of a segment of the cochlea in $\mathbf{a}$ is shown in $\mathbf{b}$. The identified SGNs in this segment is indicated by magenta circles (b'). Another method to label $\mathrm{TH}^{+} \mathrm{SGNs}$ is by 
heavy tamoxifen injection of a knock-in $T h^{2 A-C r e E R}$ mouse line crossed to reporter lines (labeling not shown). Both TH antibody staining (pre-hearing: P6-8, $\mathrm{n}=2$; hearing: 1-2 month old, $n=6$ ) and mouse line labeling (pre-hearing: $n=3$, hearing: $n=3$ ) showed an apical-to-basal gradient of numbers of SGNs labeled (c and $\left.\mathbf{c}^{\prime}\right)$. For $T h^{2 A-C r e E R}$ mouse line labeling, tamoxifen injections were administered either before or after hearing onset for the pre-hearing and hearing groups respectively. For the pre-hearing group particularly, some cochlear samples were not examined until after hearing onset to ensure a sufficient amount of time for reporter protein expression. TH antibody staining before and after hearing-onset (d) shows a similar pattern (left) and number (right) of labeled SGNs. Scale bar: $200 \mu \mathrm{m}$ in a, and $100 \mu \mathrm{m}$ in $\mathbf{b}$ and b'. Shaded areas represent standard deviations. 


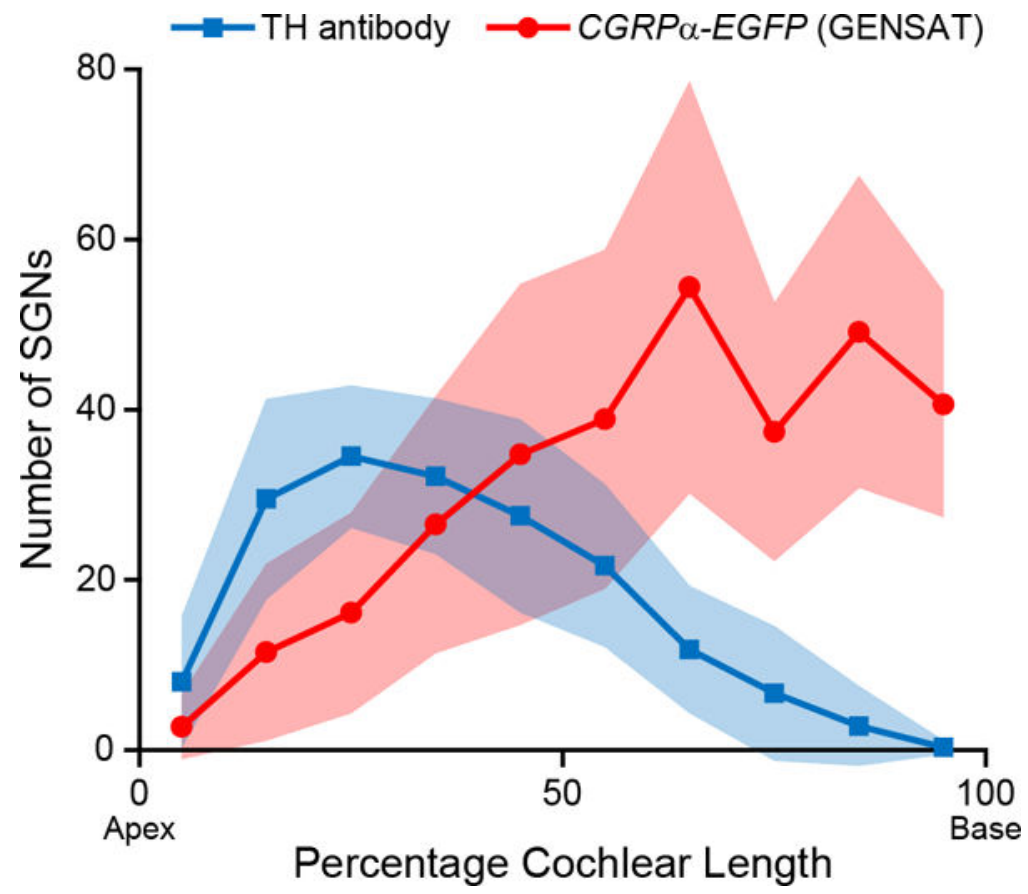

Figure 7. Opposing gene expression gradients of $\mathbf{T h}$ and Cgrp a in SGNs

A plot demonstrating the opposing gradients of $C_{g r p a}{ }^{+}$SGNs labeled by the CGRPa-EGFP (GENSAT) mouse line $(\mathrm{n}=8)$ and $\mathrm{TH}^{+}$SGNs labeled by $\mathrm{TH}$ antibody staining $(\mathrm{n}=6)$ along the cochlear spiral in hearing mice. 

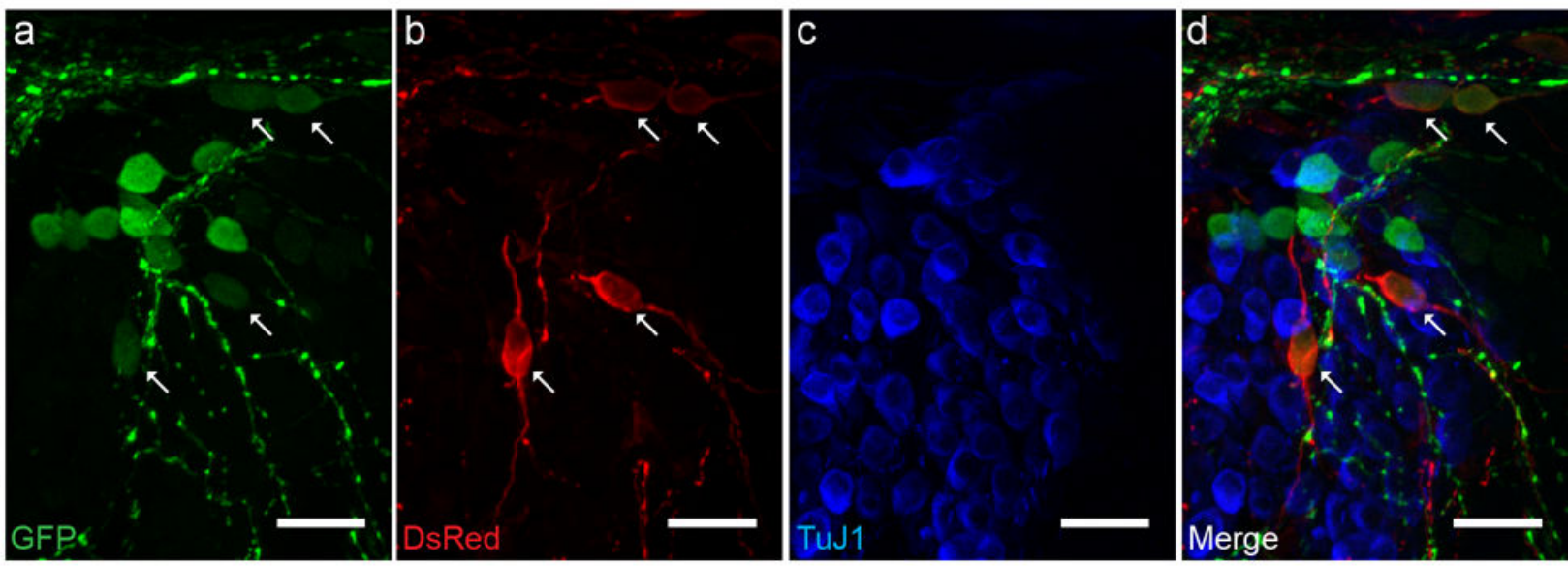

Figure 8. Co-expression of $T h$ and Cgrp $a$ can be observed in some type II afferent neurons, especially at medial turn A selected area of middle-to-basal SGN region from a one-month-old $T h^{2 A-C r e E R} ; A i 9$; $C G R P a-E G F P$ (GENSAT) mouse. Several doses of tamoxifen have been given to this mouse (four doses of 0.5-1 mg tamoxifen given after hearing onset) for the induction of tdTomato expression in $\mathrm{Th}^{+}$SGNs. SGNs co-labeled by CGRPa-EGFP(GENSAT) (a) and $T h^{2 A-C r e E R} ; A i 9$ (b) can be identified in d (arrows), suggesting that they express both Cgrpa and Th. These neurons are not stained by TuJ1 antibody (c), confirming their type II SGN identity. Scale bar: $20 \mu \mathrm{m}$.

\begin{tabular}{ll}
\hline \multicolumn{2}{l}{ Abbreviations Used in Figures } \\
\hline SGN & spiral ganglion neuron \\
\hline IHC & inner hair cell \\
\hline OHC & outer hair cell \\
\hline TH & tyrosine hydroxylase \\
\hline
\end{tabular}


Table 1

\begin{tabular}{|c|c|c|c|}
\hline Antigen & Immunogen & Manufacturer, RRID, host & Dilution \\
\hline CGRP & $\begin{array}{l}\text { Synthetic rat alpha-CGRP coupled to bovine } \\
\text { thyroglobulin (BTg) with glutaraldehyde }\end{array}$ & $\begin{array}{l}\text { ImmunoStar Cat\# 24112, RRID:AB_572217, } \\
\text { rabbit polyclonal }\end{array}$ & $1: 500-1: 1000$ \\
\hline $\mathrm{TH}$ & $\begin{array}{l}\text { purified, SDS-denatured rat pheochromocytoma } \\
\text { tyrosine hydroxylase }\end{array}$ & $\begin{array}{l}\text { Millipore Cat\# 657012-15UG, } \\
\text { RRID:AB_566341, rabbit polyclonal }\end{array}$ & $1: 500-1: 1000$ \\
\hline GFP & Purified recombinant peptide produced in E. coli. & $\begin{array}{l}\text { SICGEN Cat\# AB0020-200, RRID:AB_2333099, } \\
\text { goat polyclonal }\end{array}$ & $1: 5000$ \\
\hline DsRed & DsRed-Express protein & $\begin{array}{l}\text { Clontech Laboratories Cat\# 632496, } \\
\text { RRID:AB_10013483, Rabbit polyclonal }\end{array}$ & $1: 200-1: 1000$ \\
\hline NKAa 3 & Canine cardiac microsomes & $\begin{array}{l}\text { Thermo Fisher Scientific Cat\# MA3-915, } \\
\text { RRID:AB_2274447, mouse Monoclonal }\end{array}$ & $1: 300$ \\
\hline TuJ1 & microtubules derived from rat brain & $\begin{array}{l}\text { Covance Research Products Inc Cat\# } \\
\text { MMS-435P-250, RRID:AB_10063408, mouse } \\
\text { monoclonal }\end{array}$ & $1: 300$ \\
\hline TuJ1 & microtubules derived from rat brain & $\begin{array}{l}\text { Covance Research Products Inc Cat\# } \\
\text { MRB-435P-100, RRID:AB_10175616, rabbit } \\
\text { monoclonal }\end{array}$ & $1: 300$ \\
\hline Peripherin & $\begin{array}{l}\text { Electrophoretically pure trp-E-peripherin fusion } \\
\text { protein, containing all but the } 4 \mathrm{~N} \text { terminal amino } \\
\text { acids of rat peripherin. }\end{array}$ & $\begin{array}{c}\text { Millipore Cat\# AB1530, RRID:AB_90725, rabbit } \\
\text { polyclonal }\end{array}$ & $1: 200$ \\
\hline Myosin VI & $\begin{array}{l}\text { synthetic peptide corresponding to an epitope within } \\
\text { the C-terminal of human Myosin VI, with N-terminal } \\
\text { cysteine added, conjugated to KLH }\end{array}$ & $\begin{array}{l}\text { Sigma-Aldrich Cat\# M5187, RRID:AB_260563, } \\
\text { rabbit polyclonal }\end{array}$ & $1: 500$ \\
\hline Myosin VIIa & $\begin{array}{l}\text { Synthetic peptide containing a.a. } 927-1203 \text { of human } \\
\text { myosin VIIa protein }\end{array}$ & $\begin{array}{l}\text { DSHB Cat\# MYO7A 138-1, RRID:AB_2282417, } \\
\text { mouse monoclonal }\end{array}$ & $1: 200-1: 500$ \\
\hline VAChT & $\begin{array}{l}\text { Synthetic peptide corresponding to the C-terminus of } \\
\text { the predicted rat VAChT protein. }\end{array}$ & $\begin{array}{l}\text { Millipore Cat\# AB1588, RRID:AB_11214110, } \\
\text { guinea pig polyclonal }\end{array}$ & $1: 500-1: 1000$ \\
\hline SV2 & Purified synaptic vesicles & $\begin{array}{c}\text { DSHB Cat\# SV2, RRID:AB_2315387, mouse } \\
\text { monoclonal }\end{array}$ & $1: 300$ \\
\hline
\end{tabular}


Table 2

\begin{tabular}{|c|c|c|c|}
\hline Reactivity (isotype) & Host & Conjugate & Manufacturer, RRID \\
\hline Anti-rabbit IgG $(\mathrm{H}+\mathrm{L})$ & Donkey & Alexa Fluor 488 & Thermo Fisher Scientific Cat\# A-21206 also A21206 RRID:AB_2535792 \\
\hline Anti-rabbit IgG $(\mathrm{H}+\mathrm{L})$ & Donkey & Alexa Fluor 568 & Thermo Fisher Scientific Cat\# A10042 RRID:AB_2534017 \\
\hline Anti-mouse IgG $(\mathrm{H}+\mathrm{L})$ & Donkey & Alexa Fluor 488 & Molecular Probes Cat\# A-21202 also A21202 RRID:AB_141607 \\
\hline Anti-mouse IgG $(\mathrm{H}+\mathrm{L})$ & Donkey & Alexa Fluor 568 & Thermo Fisher Scientific Cat\# A10037 RRID:AB_2534013 \\
\hline Anti-mouse IgG $(\mathrm{H}+\mathrm{L})$ & Donkey & Alexa Fluor 647 & Molecular Probes Cat\# A-31571 also A31571 RRID:AB_162542 \\
\hline Anti-goat IgG $(\mathrm{H}+\mathrm{L})$ & Donkey & Alexa Fluor 488 & Thermo Fisher Scientific Cat\# A-11055 also A11055 RRID:AB_2534102 \\
\hline Anti-guinea pig IgG $(\mathrm{H}+\mathrm{L})$ & Donkey & Alexa Fluor 647 & Jackson ImmunoResearch Labs Cat\# 706-605-148 RRID:AB_2340476 \\
\hline
\end{tabular}

